\title{
Cytokeratin19 induced by HER2/ERK binds and stabilizes HER2 on cell membranes
}

\author{
J-h Ju ${ }^{1,7}$, S Oh $^{1,7}$, K-m Lee ${ }^{1}$, W Yang ${ }^{1}$, KS Nam ${ }^{1}$, H-G Moon ${ }^{2}$, D-Y Noh ${ }^{2}$, CG Kim ${ }^{1}$, G Park ${ }^{3}$, JB Park ${ }^{3}$, T Lee ${ }^{4}$, CL Arteaga ${ }^{5}$ and I Shin $^{\star, 1,6}$
}

Cytokeratin19 (KRT19) is widely used as a biomarker for the detection of disseminated tumors. Using an LC-MS/MS proteomics approach, we found that KRT19 was upregulated in HER2-overexpressing cells and tissues. KRT19 expression was induced by HER2-downstream ERK at the transcriptional level. Another HER2-downstream kinase, Akt, was found to phosphorylate KRT19 on Ser35 and induce membrane translocation of KRT19 and remodeling of KRT19 from filamentous to granulous form. KRT19 phosphorylated by Akt could bind HER2 on the plasma membrane and stabilized HER2 via inhibition of proteasome-mediated degradation of HER2. Silencing of KRT19 by shRNA resulted in increased ubiquitination and destabilization of HER2. Moreover, treatment of KRT19 antibody resulted in downregulation of HER2 and reduced cell viability. These data provide a new rationale for targeting HER2-positive breast cancers.

Cell Death and Differentiation (2015) 22, 665-676; doi:10.1038/cdd.2014.155; published online 24 October 2014

Overexpression of HER2 is associated with aggressive tumors and poor clinical prognosis. ${ }^{1}$ The mechanism(s) by which HER2 overexpression elicits more severe tumor phenotypes remains largely obscure; however, it has been reported that HER2 is a preferred binding partner for other HER family receptors ${ }^{2}$ and intracellular signaling from HER2containing heterodimers is more robust. ${ }^{3}$ The HER2 receptor activates several signal pathways including the phosphatidylinositol-3-kinase-PKB/Akt pathway and the extracellular signal-regulated protein kinase (ERK) pathway. ${ }^{4,5}$ Akt regulates cellular survival and metabolism via phosphorylation of many downstream effectors. ${ }^{6}$ Numerous substrates of Akt with the recognition motif of RXRXX(S/T), were reported. ${ }^{7}$ Another HER2-downstream signaling molecule, ERK is found in the cytosol of quiescent cells, but translocates to the nucleus upon activation by upstream kinases. ${ }^{8}$ Once in the nucleus, ERK can phosphorylate and regulate transcription factors, including Elk-1, ${ }^{9} \mathrm{C}$-fos, ${ }^{10}$ and Sp1. ${ }^{11}$

Cytokeratins (KRTs) are intermediate filaments found in epithelial tissue. ${ }^{12}$ KRTs are dynamically regulated and interact with a range of cellular proteins including kinases, receptors, adaptors, and other types of effector molecules to regulate cellular responses to apoptosis, cell migration, and growth. ${ }^{13}$ KRT19 is the smallest $(40 \mathrm{kDa})$ known intermediate filament protein $^{14}$ and differs from other KRTs in that it has a short tail domain. ${ }^{15}$ KRT19 is used as a marker for RT-PCR-mediated detection of tumor cells disseminated in lymph nodes, peripheral blood, and bone marrow of breast cancer patients, and its positivity could be considered as a prognostic indicator. ${ }^{16-18}$
Using a proteomics approach, two-dimensional digestLC-MS/MS, ${ }^{19}$ we confirmed that KRT19 expression is upregulated in HER2-overexpressing cells. To determine the signaling pathway responsible for upregulation of KRT19, we investigated the roles of HER2-downstream molecules such as ERK and Akt in KRT19 expression, as well as its subcellular distribution. Furthermore, we also examined the role of KRT19 in stabilizing HER2 on the cell membrane and the effect of KRT19 antibody on proliferation of HER2-positive cancer cells.

\section{Results}

HER2 expression is coupled with KRT19 expression. We found that several KRTs were upregulated in MCF-7 HER2 cells as compared with control cells by LC-MS/MS proteomics (Supplementary Table 1). We confirmed the expression of KRTs by western blot analyses (Figure 1a). KRT19 was also upregulated both at the transcriptional and translational level in high-HER2-expressing breast cancer cells (Figure 1b), suggesting that HER2 expression is coupled with KRT19 expression. We confirmed these results in vivo using a mouse model that overexpresses HER2/neu. Both immunohistochemistry and RT-PCR approaches revealed that KRT19 was upregulated in the mammary glands of MMTV-HER2/neu mice as compared with their wild-type (WT) littermates (Figure 1c). HER2 levels in human breast tumor tissue were also strongly correlated with KRT19 levels (Figure 1d). To exclude the possibility that HER2 expression increases the solubility of KRT19 rather than upregulating

\footnotetext{
${ }^{1}$ Department of Life Science, Hanyang University, Seoul, Korea; ${ }^{2}$ Cancer Research Institute, Seoul National University College of Medicine, Seoul, Korea; ${ }^{3}$ The Specific Organs Cancer Branch, Research Institute and Hospital, National Cancer Center, Goyang-si, Korea; ${ }^{4}$ NOVA Cell Technology Inc., Pohang, Korea; ${ }^{5}$ Department of Medicine, Vanderbilt University, Nashville, TN, USA and ${ }^{6}$ Natural Science Institute, Hanyang University, Seoul, Korea

*Corresponding author: I Shin, Natural Science Institute, Hanyang University, 222 Wangsimni-ro, Seongdong-gu, Seoul 133-791, Korea. Tel: +822 2220 2562; Fax: +822 2298 2562; E-mail: incheol@ hanyang.ac.kr

${ }^{7}$ These authors contributed equally to this work.

Abbreviations: KRTs, cytokeratins; KRT19, cytokeratin19; ERK, extracellular signal-regulated protein kinase; WT, wild type; TG, transgenic; HA-Akt1-CA, constitutively active Akt1; HA-Akt1-KD, kinase-dead Akt1; HER2-FL-myc, full-length HER2; HER2-ICD-myc, intracellular HER2; HER2-ECD-myc, extracellular HER2; CHX, cycloheximide; VEGF, vascular endothelial growth factor

Received 10.4.14; revised 12.8.14; accepted 29.8.14; Edited by JP Medema; published online 24.10.14
} 
a

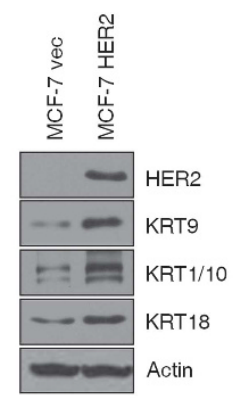

b

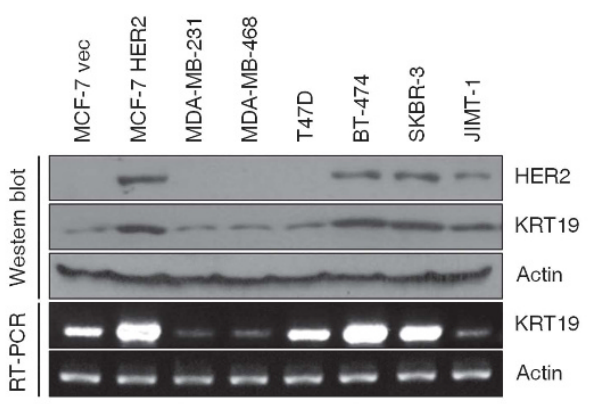

c

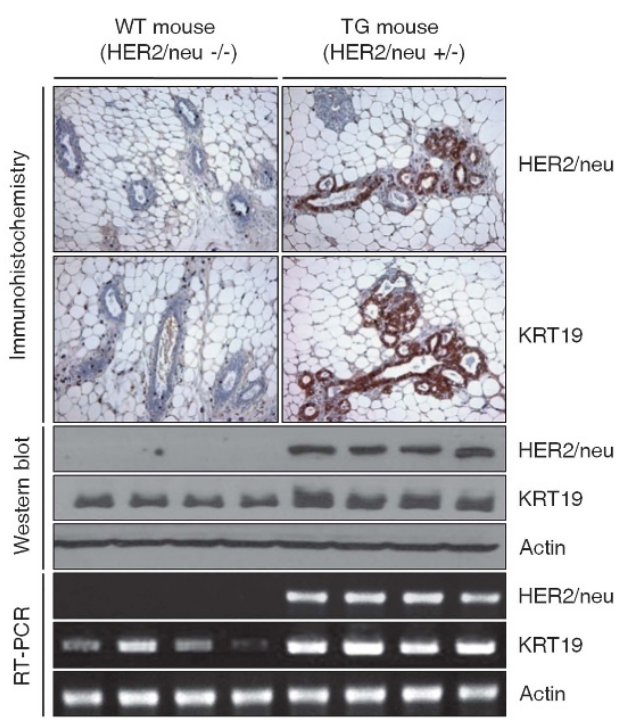

d Breast cancer tissues $(n=21)$
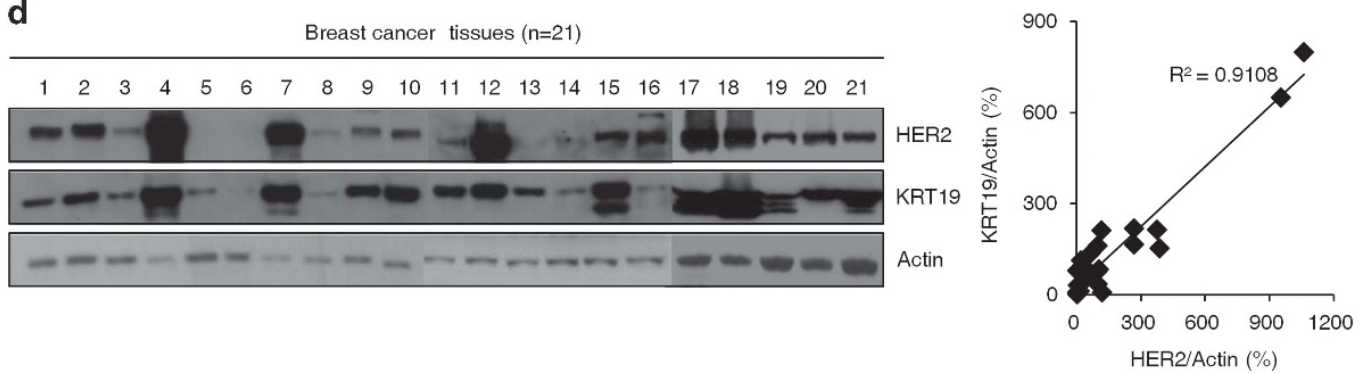

Figure 1 Expression of KRT19 is strongly correlated with HER2 levels in cultured breast cancer cells, transgenic mouse tissues, and patient tumor samples (a) Total cell lysates were prepared from MCF-7 vec and MCF-7 HER2 cells and subjected to the indicated western blot analyses with HER2, KRT9, KRT1/10 or KRT18 antibodies. Actin was used to verify equal loading. (b) Total cell lysates were prepared from human breast cancer cell lines. The samples were resolved by SDS-PAGE and subjected to western blot analyses with HER2 or KRT19 antibodies. RNA was extracted from human breast cancer cell lines and the samples were subsequently analyzed by RT-PCR using KRT19specific primers. Actin primers were used as loading controls. (c) Paraffin-embedded sections from the \#4 mammary gland tissues of MMTV-HER2/neu transgenic mice and wildtype littermates were subjected to immunohistochemistry with a KRT19 antibody. MMTV-HER2/neu transgenic mice and wild-type littermates (16 weeks, $n=4$ each) \#4 mammary gland tissues were analyzed by RT-PCR using mouse KRT19 primers, and western blot analyses with HER2 or KRT19 antibodies. (d) Western blot analysis showing expression levels of HER2 and KRT19 protein in primary tumor lysates from breast cancer patients $(n=21)$. The positive relationship between HER2 and KRT19 expression level is indicated $\left(R^{2}=0.9108\right)$

levels of KRT19 protein, both soluble fraction and insoluble pellets were tested for KRT19 expression (Supplementary Figure 1). These results indicate that HER2 expression is coupled with increased KRT19 in both detergent-soluble and -insoluble fractions.

\footnotetext{
Expression of KRT19 is modulated by HER2-downstream ERK signaling at the level of transcription. Using various inhibitors of protein kinases, we investigated whether any of the kinase activity is responsible for the upregulation of KRT19. RT-PCR analyses revealed that blockade of HER2 by ZD1839 or MEK by U0126 could decrease KRT19 mRNA in a dose-dependent manner (Figures $2 \mathrm{a}$ and $\mathrm{c}$ ). The other inhibitors failed to exert any effect on KRT19 mRNA, suggesting that upregulation of KRT19 is mediated specifically by the tyrosine kinase activity of HER2 and its downstream MEK/ERK signaling.
}

To determine whether HER2-induced upregulation of KRT19 mRNA was mediated by increased transcriptional activity of the KRT19 promoter, reporter assays were performed (Figure 2b, left panel). We used the pK19-1970LucReporter containing the promoter region of KRT19. ${ }^{20}$ The transcriptional activity of the KRT19 promoter in MCF-7 HER2 cells was significantly higher than control cells. We obtained similar results in 293T cells co-transfected with HER2 and MEK or ERK, but not by other kinases (Figure $2 b$, right panel).

As depicted in Figures 2d and e, only ZD1839 and U0126 could downregulate protein levels of KRT19 in a dosedependent fashion. These results indicate that HER2 increased KRT19 expression in ectopic HER2-overexpressing cells, as well as cells expressing endogenous HER2. The expression levels of other KRTs, which were also upregulated in MCF-7 HER2 cells (Figure 1a), were not effectively inhibited by either 
a

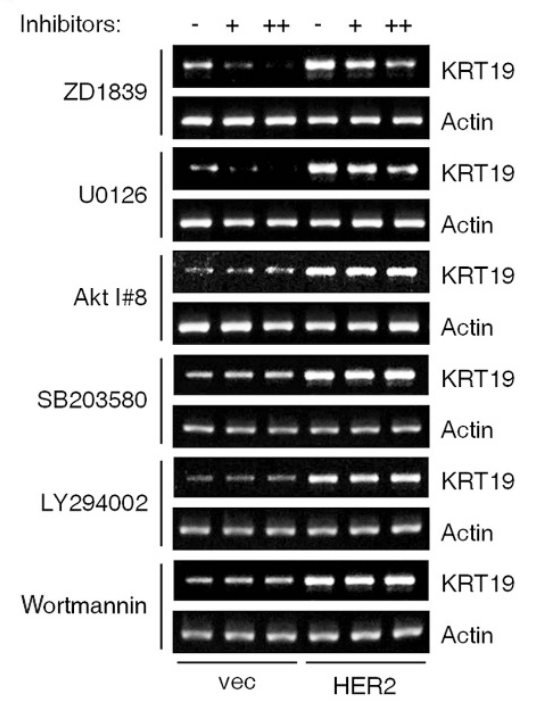

C

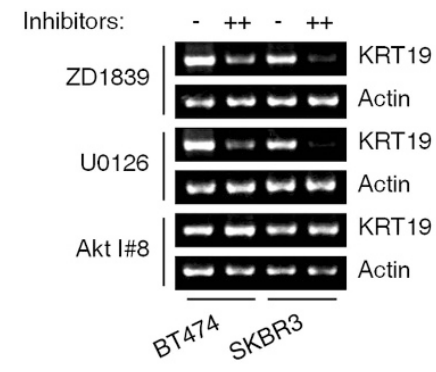

e

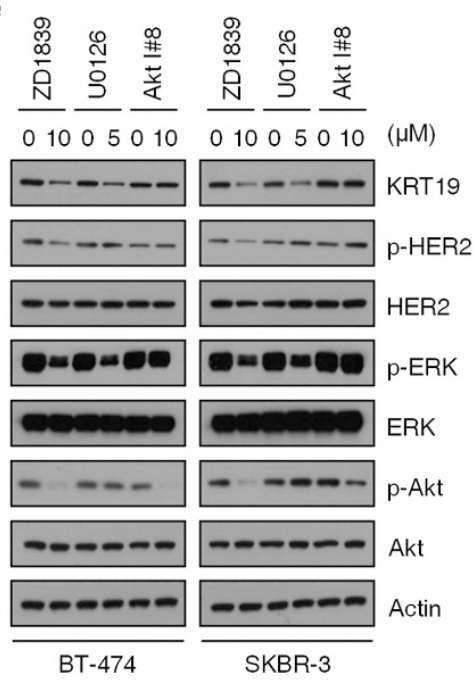

b

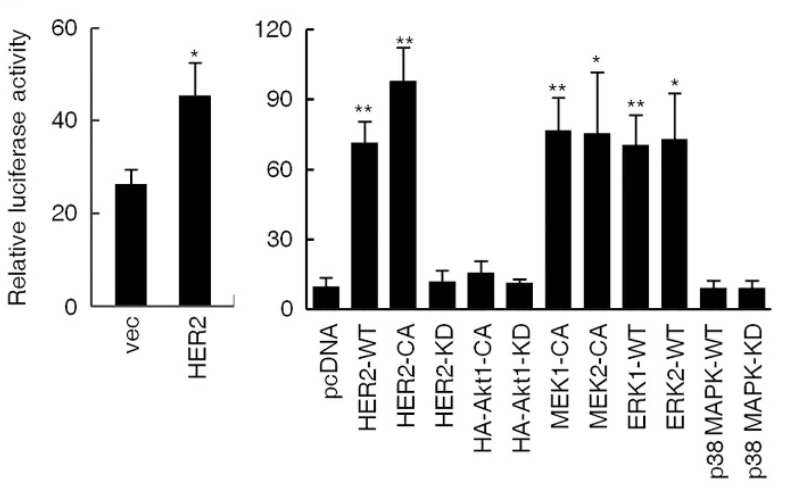

d ZD1839

$\cup 0126$

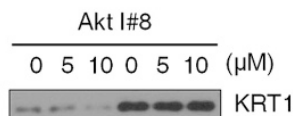

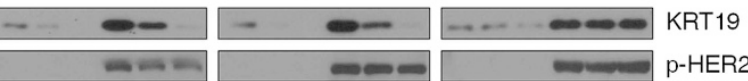

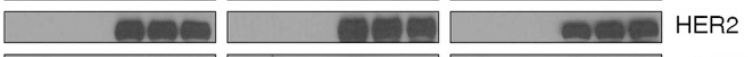

$E=\equiv=\equiv \mathrm{p}=\mathrm{ERK}$

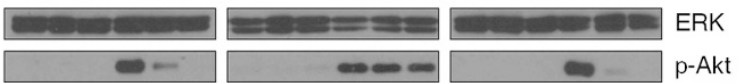

- - - - - - - - Akt

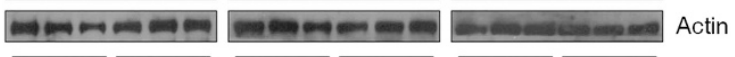

vec $\overline{\text { HER2 }} \overline{\text { vec }} \overline{\text { HER2 }} \overline{\text { vec }} \overline{\text { HER2 }}$

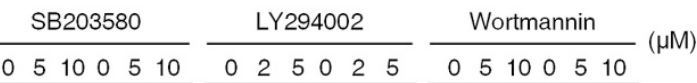

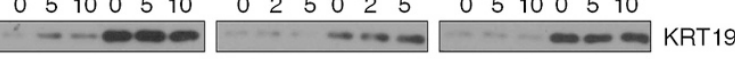

vec $\overline{\text { HER2 }} \overline{\text { vec }} \overline{\text { HER2 }} \overline{\text { vec }} \overline{\text { HER2 }}$

f

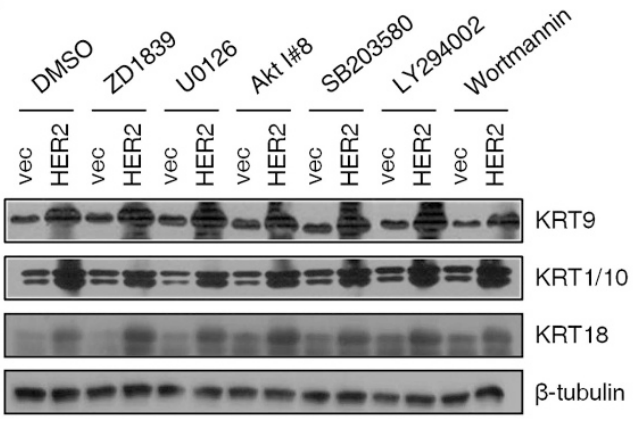

Figure 2 Expression of KRT19 is mediated by HER2 downstream of ERK at the transcriptional level (a) MCF-7 vec and MCF-7 HER2 cells were treated with kinase inhibitors $(0,5,10 \mu \mathrm{M}$ ZD1839; 0, 2, $5 \mu \mathrm{M}$ U0126; 0, 5, $10 \mu \mathrm{M}$ Akt inhibitor \#8; 0, 5, $10 \mu \mathrm{M}$ SB203580; 0, 5, $10 \mu \mathrm{M} \mathrm{LY294002} \mathrm{or} \mathrm{0,} \mathrm{2,} 5 \mu \mathrm{M}$ Wortmannin) for $24 \mathrm{~h}$. mRNA from samples were analyzed by RT-PCR. (b) MCF-7 vec and MCF-7 HER2 cells were transfected with pK19-1970-Luc reporter constructs, collected after $48 \mathrm{~h}$ and analyzed by dual luciferase assays. 293T cells were transfected with pK19-1970-Luc and pcDNA-vec, pcDNA-HER2-WT (wild type), pcDNA -HER2-CA (constitutive active), pcDNA-HER2-KD (kinase dead), pcDNA-HA-Akt1-CA, pcDNA-HA-Akt1-KD, pcDNA -MEK1-CA, pcDNA -MEK2-CA, pcDNA-ERK1-WT, pcDNA-ERK2-WT, pcDNA-p38 MAPK-WT or pcDNA-p38 MAPK-KD. After $48 \mathrm{~h}$, cells were analyzed by dual luciferase assays $\left({ }^{*} P<0.05\right.$, $\left.{ }^{\star \star} P<0.005\right)$. (c) BT-474 and SKBR-3 cells were treated with kinase inhibitors for $24 \mathrm{~h}$. mRNA from samples were analyzed by RT-PCR. (d) MCF-7 vec and MCF-7 HER2 cells were treated with kinase inhibitors for $24 \mathrm{~h}$, and the cell lysates were resolved by SDS-PAGE and subjected to western blot analyses with the indicated antibodies. (e) BT-474 and SKBR-3 cells were treated with kinase inhibitors for $24 \mathrm{~h}$. The cell lysates were subjected to western blot analyses with the indicated antibodies. (f) MCF-7 vec and MCF-7 HER2 cells were treated with kinase inhibitors for $24 \mathrm{~h}$. The cell lysates were subjected to western blot analyses with KRT9, KRT1/10 or KRT18 antibodies 
ZD1839 or U0126 (Figure 2f), indicating that HER2/MEK/ERK signaling could specifically upregulate KRT19.

\section{Akt-mediated phosphorylation of KRT19 results in remodeling of KRT19 from filamentous to granulous} forms. By using Scansite (http://scansite.mit.edu), we found two possible Akt phosphorylation sites in KRT19 (Figure 3a). Although the optimal recognition motif is $R X R X X(S / T)$, several reports indicate that $R$ at -5 position is not critically required for Akt substrates, ${ }^{21-24}$ which is interesting as the putative Akt phosphorylation sites on KRT19, S10 and S35, both lack $R$ at -5 . To determine whether one of these residues is indeed phosphorylated by Akt, WT or alaninesubstitution KRT19 mutants were transiently co-transfected with constitutively active Akt1 into 293T cells, and the cell lysates were immunoprecipitated with a KRT19 antibody. The resulting immunoprecipitate complexes were then probed with an Akt substrate antibody, which can recognize phosphorylated $\mathrm{RXX}(\mathrm{S} / \mathrm{T})$ residues. ${ }^{25}$ Immunoprecipitates containing either the KRT19-WT or KRT19-S10A were recognized by the Akt substrate antibody, whereas KRT19S35A and KRT19-S10A S35A were not immunoreactive against the antibody, suggesting that $\mathrm{S} 35$ of KRT19 may be the putative Akt phosphorylation site (Figure 3a). In MCF-7 HER2 cells that have much higher Akt activity than MCF-7 vec cells due to HER2 overexpression (Figure 2d), antiKRT19 immunoprecipitates exhibited stronger immunoreactivity toward the Akt substrate antibody (Figure 3a). To exclude the possibility that other Akt-downstream protein kinase might be involved, in vitro kinase assays were performed (Figure 3b). Our results showed that Akt could effectively phosphorylate KRT19 at S35, but not S10. Furthermore, in cells co-transfected with constitutively active Akt, only KRT19-WT and KRT19-S10A could incorporate [32P]-orthophosphate, confirming that S35 in KRT19 is an Akt phosphorylation site (Figure $3 \mathrm{c}$ ). Studies using kinase inhibitors in HER2-overexpressing cells (Figure 3d) revealed that inhibition of Akt could inhibit KRT19 phosphorylation.

It appeared that KRT19 subcellular localization and appearance were vastly different between MCF-7 vec and MCF-7 HER2 cells (Figure 3e). In MCF-7 vec cells, KRT19 exhibited a filamentous shape, whereas KRT19 in MCF-7 HER2 cells have a granulated morphology. To determine whether phosphorylation of S35 is responsible for the modification of KRT19, we co-transfected KRT19 with constitutively active Akt (HA-Akt1-CA), kinase-dead Akt (HA-Akt1-KD) or other kinases into 293T cells. In cells co-transfected with KRT19-WT or KRT19-S10A with HA-Akt1-CA, HER2-WT or HER2-CA, immunostaining patterns of KRT19 were similar to that of KRT19 in MCF-7 HER2 cells, whereby KRT19 molecules in the cytosol have a granulated appearance (Supplementary Figure 2). In contrast, the staining patterns of KRT19 in cells co-transfected with other kinase constructs and KRT19-S35A or KRT19-S10A S35A were very similar to MCF-7 vec cells, whereby KRT19 appeared as a long filamentous fiber (Supplementary Figure 2). Quantification of the results in Figure 3e and Supplementary Figure 2 is shown in Figures $3 \mathrm{f}$ and $\mathrm{g}$. These results indicated that Akt-mediated phosphorylation of KRT19 on S35 was responsible for the KRT19 subcellular shape.
Physical association of HER2 and KRT19 in the plasma membrane. We performed immunofluorescence staining using HER2 and KRT19 antibodies. As depicted in optically sectioned images (Supplementary Figure 3a), a significant portion of KRT19 was identified in the cell periphery. Membrane KRT19 appeared to be colocalized with HER2 in the cell membrane. As it was previously documented that KRTs are mostly cytosolic, we reconfirmed membrane localization of KRT19 by other methods including subcellular fractionation, cell-surface immunofluorescence labeling and cell-surface protein biotinylation assay. Membrane fractions of MCF-7 HER2 cells contained KRT19, whereas that from MCF-7 vec cells did not (Figure 4a). Similarly, BT-474 cells with high HER2 also contained KRT19 in the membrane. Aquaporin 4 was a membrane fraction marker. Cell surface immunofluorescence labeling confirmed that immunoreactivity toward KRT19 antibody was found in the membranes of MCF-7 HER2 and BT-474 cells, but not in membranes of MCF-7 vec cells (Figure 4c). The cell-surface protein biotinylation assay also confirmed that membrane KRT19 was upregulated in MCF-7 HER2 cells as compared with vec cells (Figure $4 \mathrm{~d}$, left panels, lane 1 versus 3 ). After serum starvation, membrane KRT19 was decreased (lanes 5 and 6) and treatment with insulin-like growth factor could rescue the levels (lanes 7 and 8). Akt inhibitor could also decrease membrane KRT19 (lanes 9 and 10). Essentially the same results were observed in BT-474 and SKBR-3 cells (Figure 4d, right panels). The HER2 was used as a positive control for membrane protein. Cytosolic ERK was not detected by both cell-surface-labeling experiments (Figures $4 \mathrm{c}$ and $\mathrm{d}$, right panels). We analyzed the amino-acid sequence of KRT19 by the membrane protein explorer program (http://blanco.biomol.uci.edu/mpex/). The prediction results indicated that KRT19 may contain some membraneassociated $\beta$-barrel motifs, indicating that some domain of KRT19 might be associated with the plasma membrane.

We next evaluated the possibility of a physical association of KRT19 with HER2. HER2 and KRT19 were co-immunoprecipitated in HER2-overexpressing cells (Figure 4b). When KRT19-WT was co-transfected with fulllength HER2 (HER2-FL-myc) or truncated mutant constructs (intracellular, HER2-ICD-myc or extracellular domain, HER2ECD-myc), we observed association between KRT19 and extracellular domain of HER2 (Figure 4e). We next asked whether membrane localization of KRT19 requires the presence of HER2. When HA-Akt1-CA was co-transfected with HER2, we observed membrane localization of KRT19WT and KRT19-S10A (Supplementary Figure 3b, left panels). However, when the Akt activity was blocked by HA-Akt1-KD, we could not detect any membrane-associated KRT19-WT and KRT19-S10A. Indeed, both KRT19-S35A and KRT19S10A S35A failed to exhibit membrane localization under any conditions, suggesting that both Akt-mediated phosphorylation of KRT19 on S35 and the presence of HER2 are required for the membrane localization of KRT19. These data coincide with results in Figure 4d where membrane KRT19 was decreased by the Akt inhibitor. Furthermore, it appeared that the kinase activity of HER2 was not directly required for the membrane localization of KRT19, as HER2-KD-expressing cells could also induce membrane localization of KRT19 as 
a

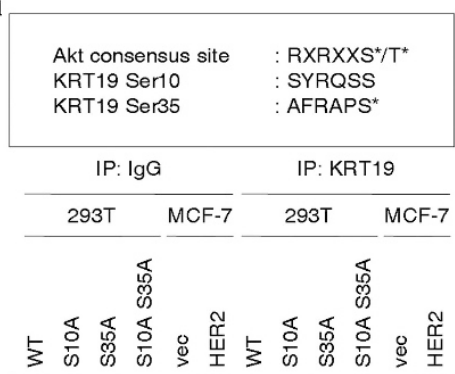

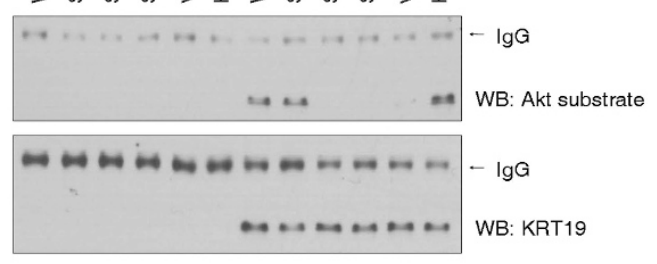

b

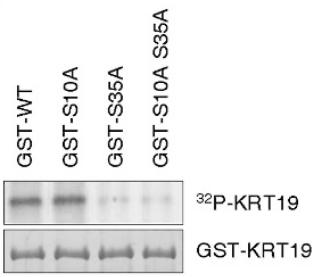

c

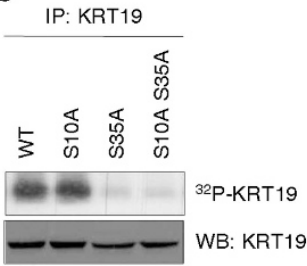

d

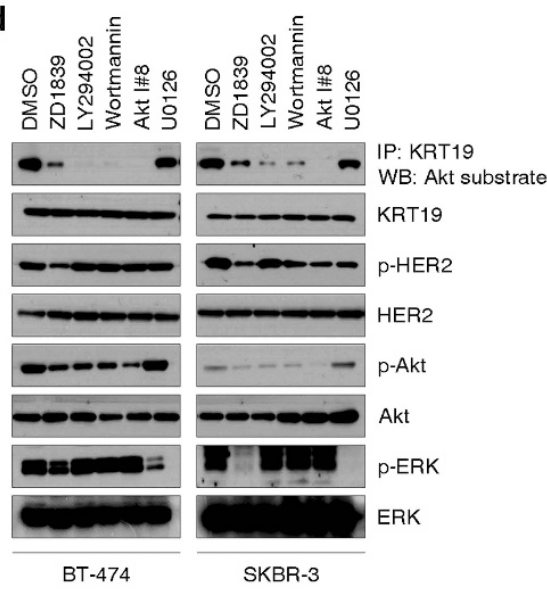

e

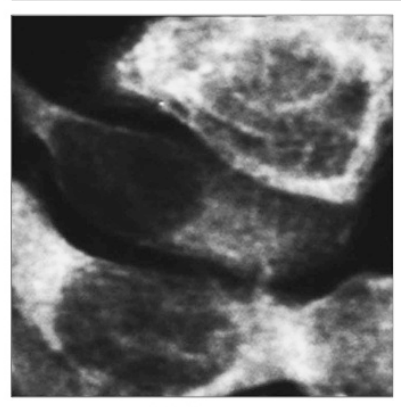

MCF-7 vec

KRT19

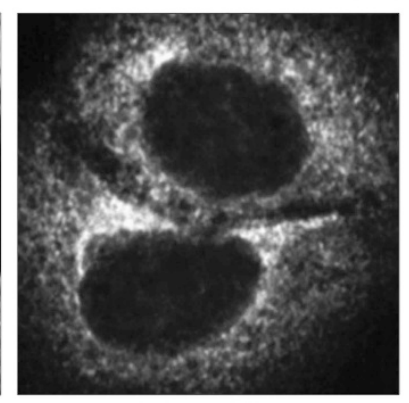

MCF-7 HER2 f

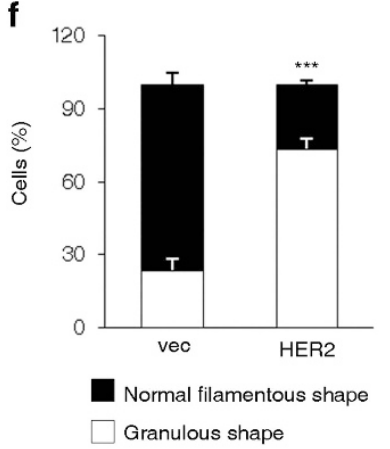

g
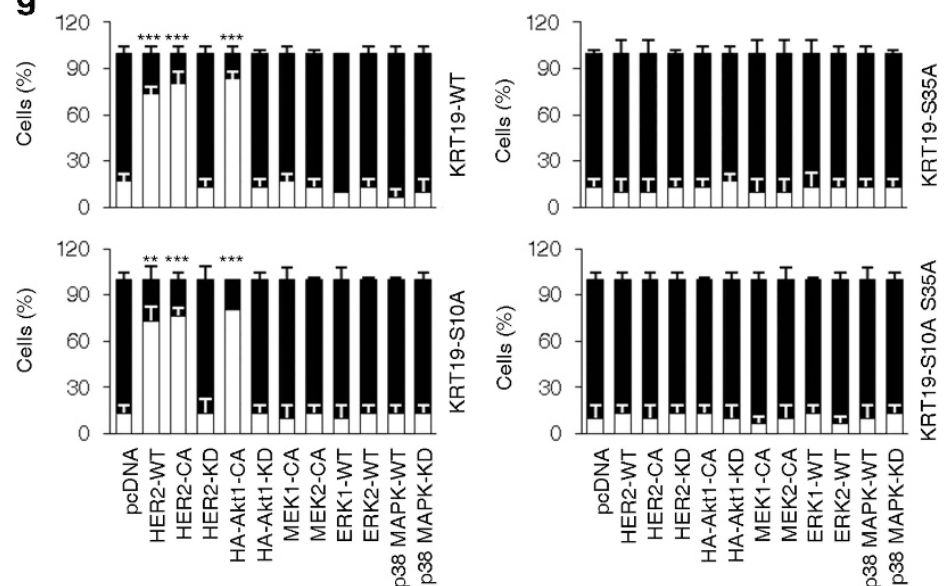

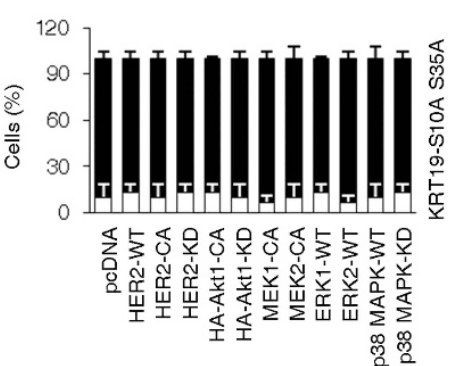

Figure 3 KRT19 is phosphorylated by HER2 downstream of Akt at Ser35, resulting in remodeling of KRT19 from filamentous to granulous form (a) Total cell lysates were incubated with KRT19 antibody overnight. Immunoprecipitates were subjected to western blot analysis with Akt substrate antibody or KRT19 antibody. (b) GST-KRT19 was prepared as described. HA-Akt precipitates were used as a kinase source for in vitro kinase assays. After the reaction, the samples were subjected to SDS-PAGE and subsequent autoradiography. Equal loading of KRT19 per reaction was confirmed by Coomassie brilliant blue staining. (c) In vivo phosphorylation of KRT19 was assessed by labeling $293 \mathrm{~T}$ cells transfected with KRT19 with 32P-orthophosphate. 32P-labeled KRT19 was precipitated with KRT19 antibody and analyzed by autoradiography. To normalize for transfection sufficiency, precipitates were subjected to KRT19 immunoblotting. (d) BT-474 and SKBR-3 cells were treated with various kinase inhibitors. The cell lysates were then subjected to immunoprecipitation and western blotting. (e) KRT19 shape in MCF-7 cells was monitored by immunostaining. MCF-7 vec and MCF-7 HER2 cells were fixed and stained with KRT19 antibody. (f) Quantification of the results in e. More than 100 cells per each independent microscopic field were observed and counted in triplicate $\left({ }^{* *} P<0.0005\right)$. (g) Quantification of the results in Supplementary Figure 2. More than 100 stained cells per each independent microscopic field were observed and counted in triplicate $\left({ }^{* \star} P<0.005,{ }^{* * *} P<0.0005\right)$ 
a

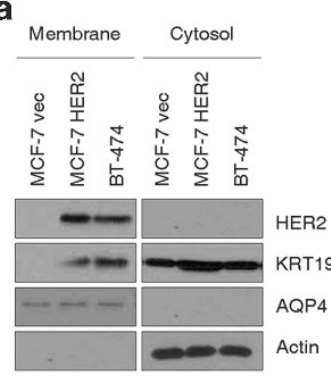

b

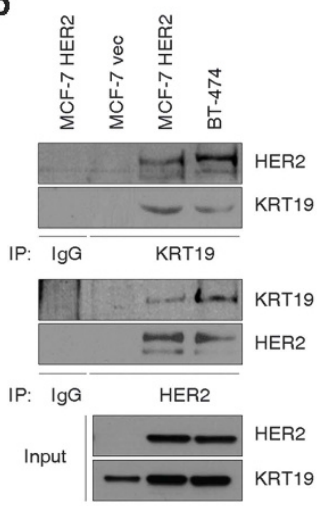

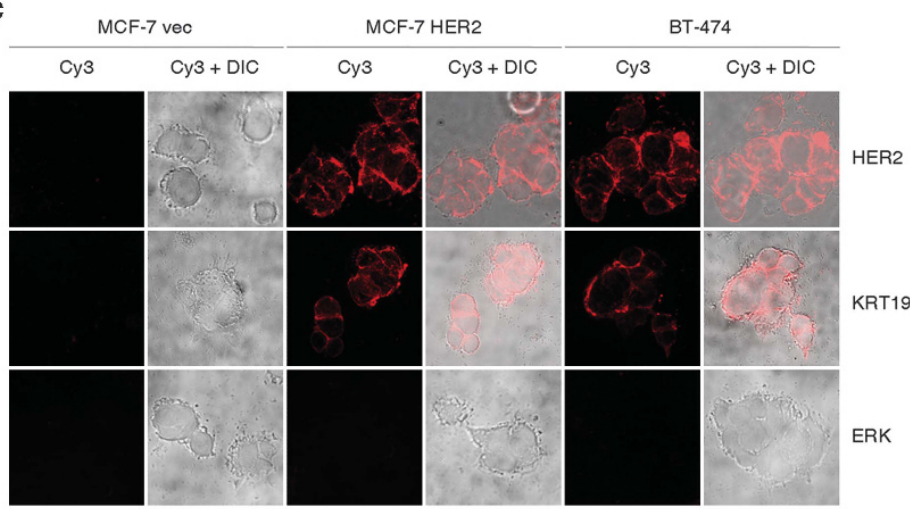

d

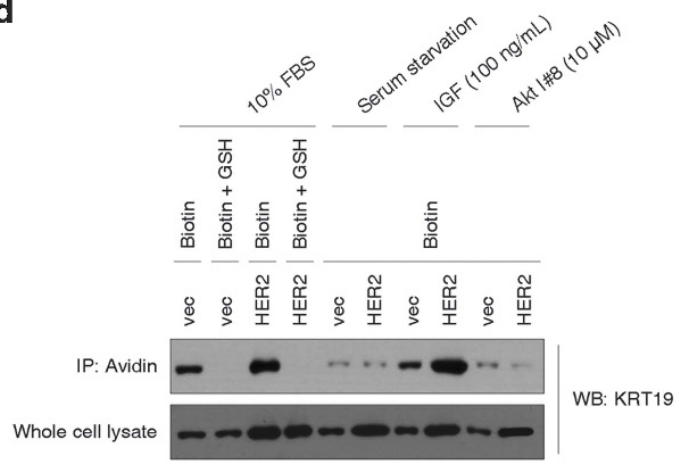

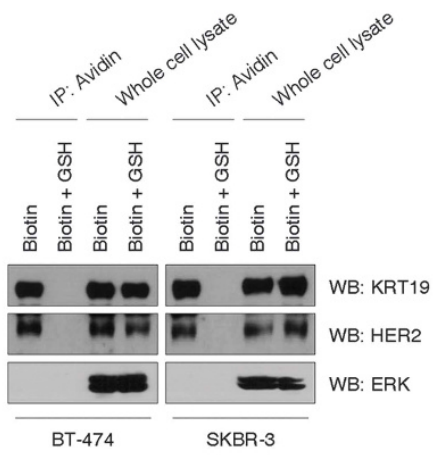

e ++++++++ KRT19-WT $+\cdots+\cdots+\cdots$ HER2-FL-myc $+\cdots+\cdots+\cdots$ HER2-ECD-myo $\cdots+\cdots+\cdots+$ HER2-ICD-myc

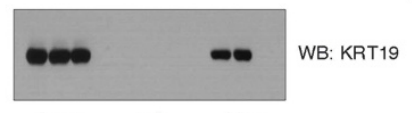
Input $\quad \lg G \quad M y c$

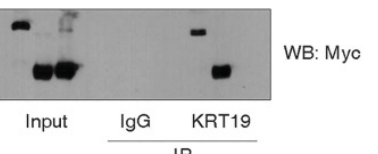

Figure 4 KRT19 is physically associated with HER2 in the plasma membrane and HER2/Akt-induced phosphorylation of KRT19 at Ser35 can induce membrane localization of KRT19 (a) MCF-7 vec, MCF-7 HER2 and BT-474 cells were fractionated into membrane and cytosol fractions. The resulting fractions were subjected to western blot analysis with HER2, KRT19, AQP4 (aquaporin4) (as membrane marker) or actin (as cytosol marker) antibodies. (b) MCF-7 HER2 and BT-474 cell lysates were incubated with HER2 or KRT19 antibodies overnight. Immunoprecipitates were subjected to western blot analysis with KRT19 or HER2 antibodies. (c) Cell surface labeling of KRT19 was conducted by cell surface immunostaining. ERK was used as an endogenous negative control protein. (d) Cell surface labeling of KRT19 was conducted by the cell surface biotinylation assay. The labeled proteins were precipitated by streptavidin and analyzed by western blot with KRT19, HER2 and ERK antibodies. ERK was used as an endogenous negative control protein. (e) KRT19 constructs were co-transfected with HER2 full-length or truncated mutant constructs in 293T cells. After $48 \mathrm{~h}$, cells were lysed and the supernatant was incubated with KRT19 or myc antibodies and protein A sepharose. The immunoprecipitates were analyzed by western blot with myc or KRT19 antibodies

long as HA-Akt1-CA was co-expressed. The requirement for the presence of HER2 and Akt activity for membrane localization of KRT19 was also confirmed by subcellular fractionation assays (Supplementary Figure 3b, right panels).

KRT19 stabilizes HER2 on cell membranes. We next asked whether HER2 and Akt-induced membrane-localized KRT19 had any effect on HER2 in the plasma membrane. Cycloheximide-decay assays showed that co-expression of KRT19-WT and KRT19-S10A significantly increased the stability of HER2-WT, whereas KRT19-S35A and KRT19S10A S35A had no effect (Figure 5a, left panel). Similarly, HA-Akt1-CA effectively upregulated the stability of HER2-KD when co-transfected with KRT19-WT or KRT19-S10A (Figure 5a, middle panel). However, when HA-Akt1-KD was co-transfected, none of the KRT19 constructs were able to increase the stability of HER2-KD (Figure 5a, right panel). These data suggest that upon phosphorylation by Akt on S35, KRT19 localizes to the cell membrane where it binds to and stabilizes HER2 in a HER2 kinase-activity-independent fashion.

To further confirm the effect of KRT19 on HER2 protein stability, KRT19 knockdown cell lines were generated. KRT19 shRNA-expressing cells exhibited a clear KRT19 knockdown phenotype as compared with control shRNA-expressing cells (Figure 5b). Knockdown of KRT19 impaired the stability of HER2 (Figure 5b, lanes 1-4 in all panels). To check whether HER2 stability conferred by presence of KRT19 was also dependent on Akt activity, an Akt inhibitor was co-treated with cycloheximide (Figure 5b, lanes 9-12 in all panels). When Akt activity was blocked, the decay slopes of HER2 were similar between control and KRT19 shRNA cells. Comparison of HER2 protein stability between control cells treated with cycloheximide alone or cycloheximide and the Akt inhibitor (Figure 5b, lanes 1-4 versus lanes 9-12 in control shRNA panels) revealed that there was a significant decrease in HER2 stability in cells treated with both the Akt inhibitor and cycloheximide compared with cells treated with cycloheximide alone. However, when the samples from KRT19 shRNA cells were compared, there were no significant differences in HER2 stability between cells treated with both the Akt inhibitor and cycloheximide compared with cells treated with cycloheximide alone (Figure 5b, lanes 1-4 versus lanes 9-12 in KRT19 shRNA panels). These data indicate that KRT19 regulates HER2 stability in an Akt-activity-dependent manner. We also checked whether inhibition of Akt activity alone could impair HER2 levels. When Akt inhibitor-treated control shRNA cells and Akt inhibitor-treated KRT19 shRNA cells were compared 
a

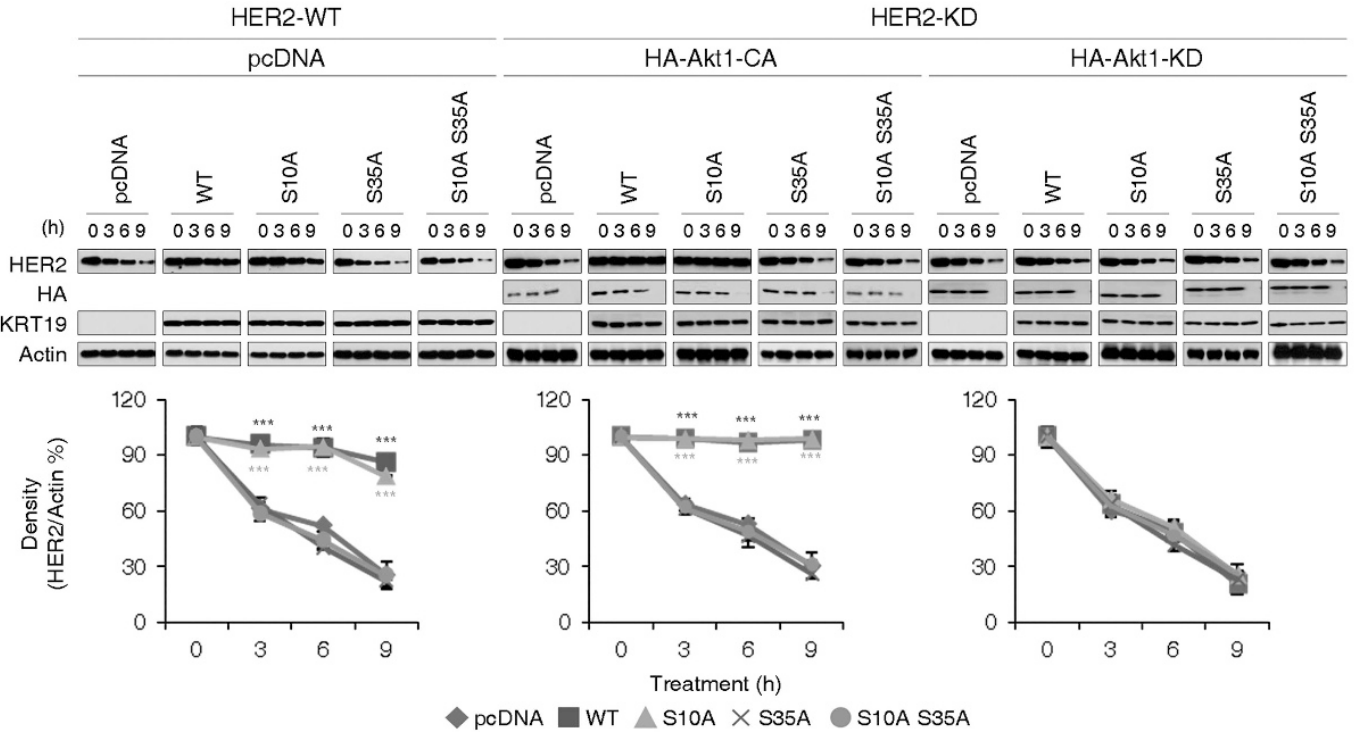

b

\begin{tabular}{|c|c|c|c|c|c|c|c|c|c|c|c|c|c|c|c|c|c|}
\hline \multicolumn{6}{|c|}{ MCF-7 HER2 } & \multicolumn{6}{|c|}{ BT-474 } & \multicolumn{6}{|c|}{ SKBR-3 } \\
\hline \multicolumn{3}{|c|}{ Cont sh } & \multicolumn{3}{|c|}{ KRT19 sh } & \multicolumn{3}{|c|}{ Cont sh } & \multicolumn{3}{|c|}{ KRT19 sh } & \multicolumn{3}{|c|}{ Cont sh } & \multicolumn{3}{|c|}{ KRT19 sh } \\
\hline 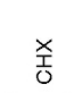 & 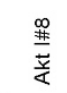 & 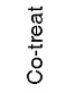 & $\underset{\text { 종 }}{\text { X }}$ & 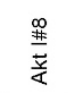 & 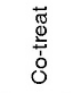 & 종 & $\begin{array}{l}\frac{\infty}{\#} \\
\frac{\#}{\#} \\
\frac{\vec{r}}{<}\end{array}$ & 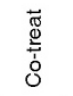 & 岸 & 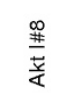 & 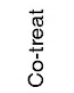 & $\underset{\substack{x \\
0}}{x}$ & 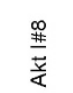 & 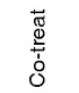 & $\underset{\substack{x \\
0}}{x}$ & 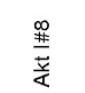 & 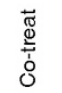 \\
\hline \multirow{2}{*}{$\overline{0369}$} & 0369 & 0369 & 0369 & 0369 & 0369 & 0369 & 0369 & 0369 & 0369 & 0369 & 0369 & 0369 & 0369 & 0369 & \multicolumn{3}{|c|}{$\overline{0369} \overline{0369} \overline{0369}$} \\
\hline & & & \multirow{2}{*}{\multicolumn{3}{|c|}{--}} & \multicolumn{3}{|c|}{ 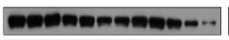 } & $-\cdots$. & $---\infty$ & --- & \multicolumn{3}{|c|}{ 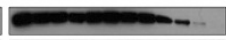 } & \multicolumn{3}{|c|}{ na nandon } \\
\hline \multicolumn{3}{|c|}{$-\cdots$} & & & & \multicolumn{3}{|c|}{ 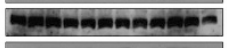 } & & & & \multirow{2}{*}{\multicolumn{3}{|c|}{ 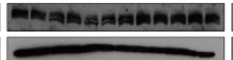 }} & \multicolumn{3}{|c|}{ 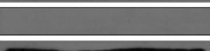 } \\
\hline \multicolumn{3}{|c|}{ - } & \multicolumn{3}{|c|}{ 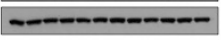 } & & & & & & & & & & & & \\
\hline
\end{tabular}

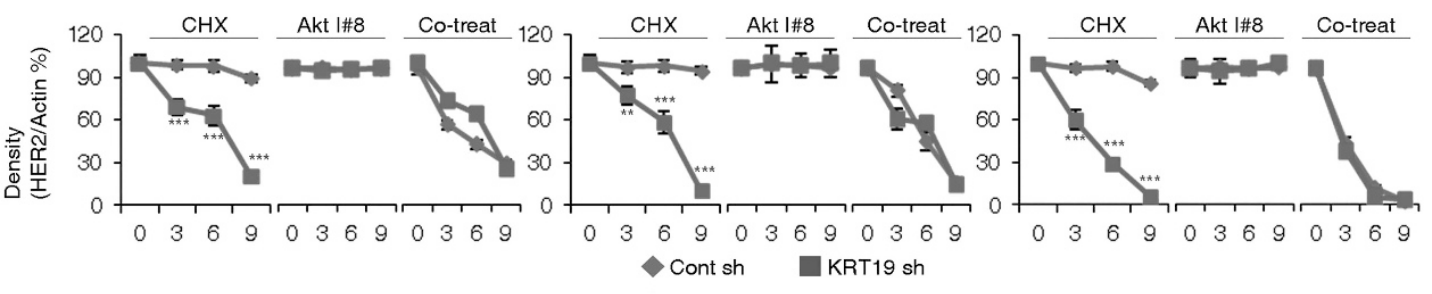

C

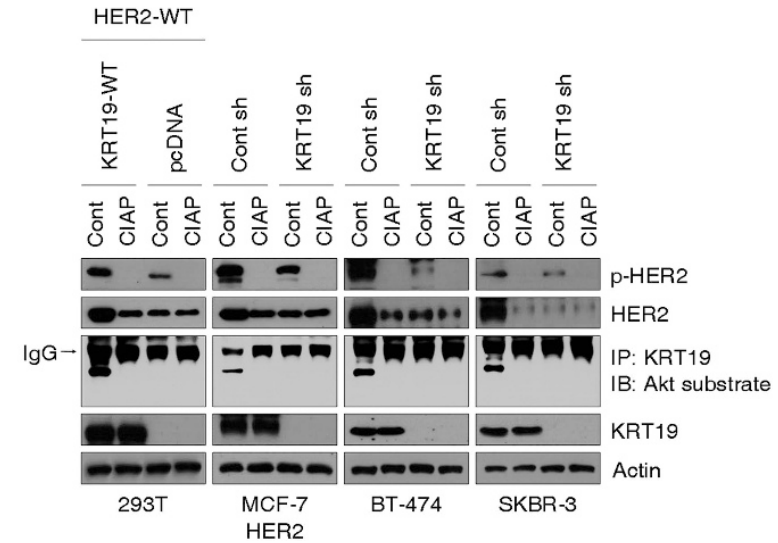

d

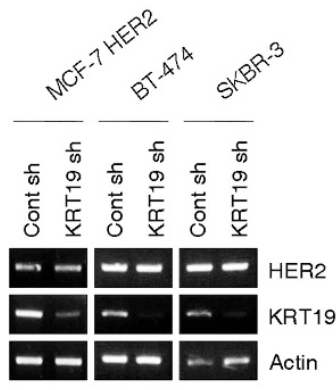

Figure 5 HER2/Akt-induced phosphorylation of KRT19 at Ser35 is essential for maintaining HER2 stability at the membrane (a) We transfected 293T cells with various combinations of HER2, Akt and KRT19 as indicated. At $48 \mathrm{~h}$ post transfection, $20 \mu \mathrm{g} / \mathrm{ml} \mathrm{CHX}$ was treated to block further protein synthesis. Cells were collected at each of the indicated time points and subjected to western blot analysis. The intensity of the bands was quantified using 1DScan software (CSP Inc, Billerica, MA, USA) and plotted as time versus ratio of HER2/actin intensity ( ${ }^{\star \star *} P<0.0005$ ). (b) Control shRNA and KRT19 shRNA cells were treated with $\mathrm{CHX}$ at $100 \mu \mathrm{g} / \mathrm{ml}$. The cell lysates were obtained at the indicated time points and prepared for western blot analysis. The intensity of the bands was quantified using 1DScan software and plotted as time versus the ratio of $\mathrm{HER} 2 /$ actin intensity for MCF-7 HER2 control shRNA and KRT19 shRNA cells $\left({ }^{\star *} P<0.005,{ }^{* \star *} P<0.0005\right)$. (c) Lysates from control shRNA, KRT19 shRNA cells, $293 T$ cells expressing KRT19 and control 293T cells were prepared. The cell lysates were treated with 20 units calf intestinal alkaline phosphatase (CIAP) per mg of protein for $1 \mathrm{~h}$ at $37^{\circ} \mathrm{C}$ to dephosphorylate phosphoproteins. (d) RNA was extracted from control shRNA and KRT19 shRNA cells using Trizol reagent. RT-PCR was performed using HER2-, KRT19-, and actin-specific primers 
(Figure 5b, 5-8 lanes in all panels), treatment with the Akt inhibitor alone did not alter HER2 stability in both control shRNA and KRT19 shRNA cells within the window of treatment times employed for this study.

When the cell lysates were treated with alkaline phosphatase to dephosphoryate phosphoproteins, we observed that HER2 levels were downregulated in cells expressing KRT19 (Figure 5c). However, in cells not expressing KRT19, treatment with alkaline phosphatase did not significantly affect HER2 protein levels. The results may suggest that the phosphorylation of KRT19 is critical in maintaining HER2 protein stability, because cell lines devoid of KRT19 do not exhibit significant differences in HER2 protein levels with or without treatment with phosphatases. Finally, to rule out the possibility of KRT19 modulating HER2 protein by inducing changes in HER2 mRNA, RT-PCR experiments were employed (Figure 5d). HER2 mRNA levels were similar both in control shRNA and KRT19 shRNA cells.

\section{KRT19 inhibits ubiquitin-mediated degradation of HER2.} We were next interested in determining whether KRT19 increases the stability of HER2 by modulation of a HER2protein-degradation mechanism. Treatment with MG132, a 26S proteasome inhibitor, resulted in increased HER2 protein level, as well as increased ubiquitination of HER2 (Figures $6 a$ and b). Increased ubiquitination of HER2 was more evident in KRT19 shRNA cells (Figure 6a lane 10 versus 12, Figure $6 \mathrm{~b}$ lane 6 versus 8 ), suggesting that a

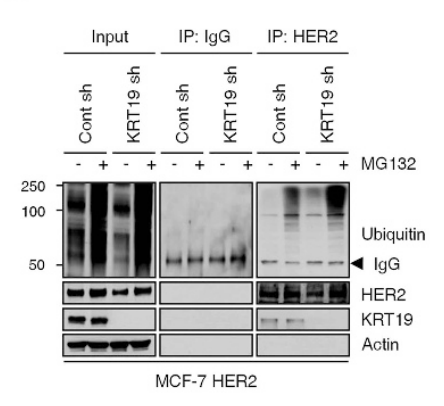

b

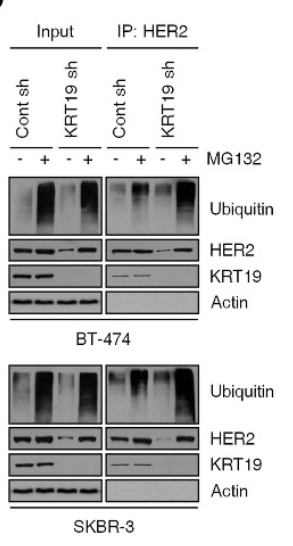

C

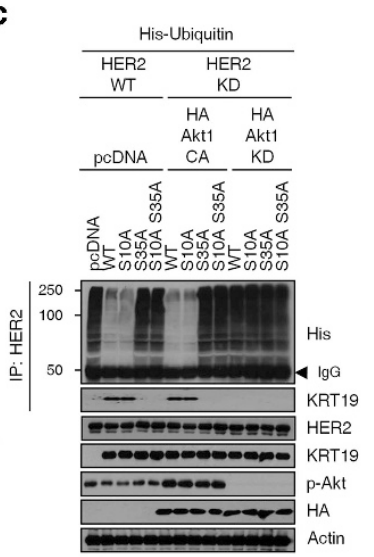

g

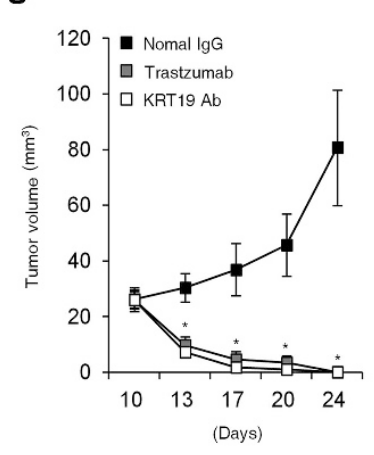

d

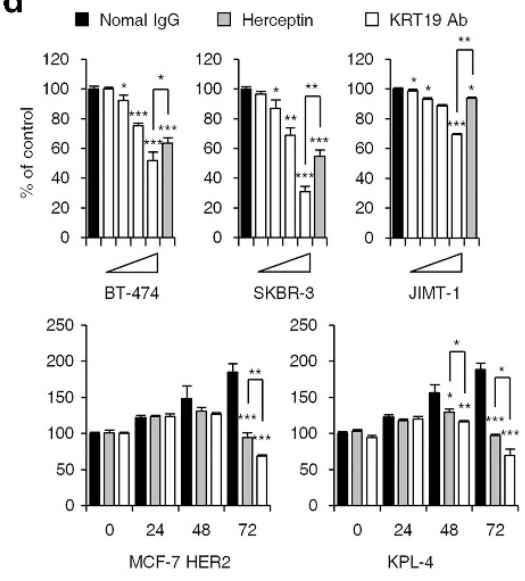

h

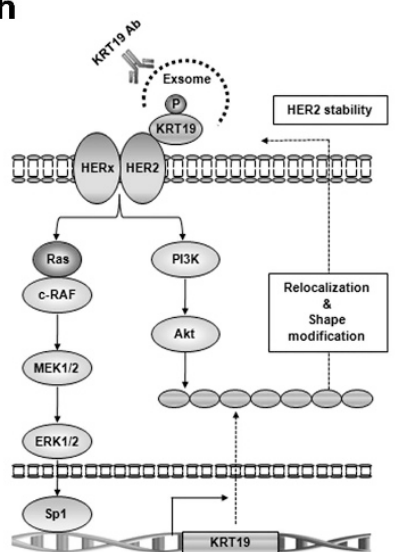

Figure 6 KRT19 inhibits ubiquitin-mediated degradation of HER2, and treatment with KRT19 antibody induces a decrease in cell proliferation via HER2 downregulation (a) MCF-7 HER2 Control shRNA and MCF-7 HER2 KRT19 shRNA cells were treated with MG132 at the concentration of $10 \mu \mathrm{M}$ for $8 \mathrm{~h}$ and lysed. The supernatant was incubated with HER2 antibody and protein A sepharose. The immunoprecipitates were analyzed by western blot with each of the indicated antibodies. (b) BT-474 control shRNA, KRT19 shRNA cells, SKBR-3 control shRNA and KRT19 shRNA cells were treated with MG132, $10 \mu \mathrm{M}$ for $8 \mathrm{~h}$ and lysed. The supernatant was incubated with HER2 antibody and protein A sepharose. The immunoprecipitates were analyzed by western blot with each of the indicated antibodies. (c) 293T cells were co-transfected with a His-Ubiquitin expression construct with the indicated plasmids. The cells were then treated with MG132 at $10 \mu \mathrm{M}$ for $8 \mathrm{~h}$ and lysed. The supernatant was incubated with HER2 antibody and protein A sepharose. The immunoprecipitates were analyzed by western blots with each of the indicated antibodies. (d) BT-474, SKBR-3, JIMT-1 (0, 5, 10, $20 \mu \mathrm{g} / \mathrm{ml})$, MCF-7 HER2 and KPL-4 cells $(20 \mu \mathrm{g} / \mathrm{ml})$ were treated with KRT19 antibody. Normal IgG and Trastzumab were treated at a dose of $20 \mu \mathrm{g} / \mathrm{ml}$. After $72 \mathrm{~h}$, cell viability was assessed by the proliferation assay. The value from normal lgG-treated cells was set to $100 \%$ and relative decreases in cell viability after KRT19 antibodies treatment were expressed as a percent of the control $\left({ }^{*} P<0.05,{ }^{* *} P<0.005,{ }^{* \star *} P<0.0005\right)$. (e) BT-474, SKBR-3 and KPL-4 cells were treated with $20 \mu \mathrm{g} / \mathrm{ml} \mathrm{KRT19}$ antibodies for $24 \mathrm{~h}$ and lysed. The samples were resolved by SDS-PAGE and subjected to western blot analyses with indicated antibodies. (f) BT-474 and SKBR-3 cells were treated with $20 \mu \mathrm{g} / \mathrm{ml} \mathrm{KRT19}$ antibodies and $10 \mu \mathrm{M} \mathrm{MG132} \mathrm{for} 24 \mathrm{~h}$ and lysed. The supernatant was incubated with HER2 antibody and protein A sepharose. The immunoprecipitates were analyzed by western blot with each of the indicated antibodies. (g) HER2-positive KPL-4 cells were injected in 8-week-old BALB/C nude mice by subcutaneous injection of $5 \times 10^{6}$ cells into both sides of the lower flank of mice ( $n=5$ per group). At day 10 after inoculation, normal lgG, Trastzumab or KRT19 antibodies were i.p. injected at the dose of $20 \mathrm{mg} / \mathrm{kg}\left({ }^{*} P<0.05\right)$. (h) A schematic model showing the mechanism of stabilization of HER2-induced KRT19 on the cell membrane. KRT19 binds HER2 via Akt-mediated phosphorylation in a Ser35-dependent manner and stabilizes HER2 on the cell membrane 
ubiquitination of HER2 is more actively processed in KRT19silenced cells. To further determine whether the phosphorylation status of KRT19 is responsible for the ubiquitination of HER2, KRT19 point mutants were co-transfected along with various combinations of kinases (Figure $6 \mathrm{c}$ ). Cells cotransfected with HER2-WT and KRT19-S35A, KRT19-S10A S35A or empty vector exhibited intensive levels of HER2 ubiquitination upon MG132 treatment (lanes 1, 4, 5). When HER2-KD was co-transfected with HA-Akt1-CA and KRT19 constructs, we observed that the pattern of HER2 ubiquitination was highly similar to that observed in HER2-WTexpressing cells (lanes $2-5$ versus $6-9$ ), suggesting that Akt activity and the S35 residue of KRT19 are absolute requirements for preventing hyper-ubiquitination of HER2. In cells co-transfected HER2-KD, HA-Akt1-KD and KRT19, all of the samples exhibited intensive ubiquitination of HER2, confirming that Akt kinase activity is necessary to prevent hyper-ubiquitination of HER2. Accordingly, KRT19 was shown to be co-immunoprecipitated with HER2 in samples exhibiting basal levels of HER2 ubiquitination (lanes 2, 3, 6, 7).

KRT19 antibody treatment inhibited proliferation of HER2-expressing cells. A dosage and time-dependent decrease in proliferation of HER2-positive cells were observed by KRT19 antibody treatment (Figure 6d). Trastzumab a therapeutic antibody against HER2 was used as a positive control. KRT19 antibody could also affect the growth of Trastzumab-resistant JIMT-1 cells. Treatment with KRT19 antibody could downregulate HER2 level and its downstream signaling in HER2-positive breast cancer cells (Figure 6e), via increased ubiquitination and subsequent degradation of HER2 (Figure 6f). We performed xenograft experiments to determine the efficiency of KRT19 antibody in vivo (Figure $6 \mathrm{~g}$ and Supplementary Figure 4). The mice were treated with KRT19 antibody or Trastzumab for 3 weeks. We found that treatment with KRT19 antibody inhibited the in vivo tumor formation. Complete tumor dissipation was observed in all mice treated with KRT19 antibody or Trastzumab at day 24. Administration of KRT19 antibody was non-toxic, as analyzed by animal survival and weight-loss analyses. These results indicate KRT19 may be a novel therapeutic target in HER2expressing cancer cells.

\section{Discussion}

We showed here that (1) expression of KRT19 was upregulated by the HER2-downstream MEK/ERK pathway, (2) HER2-downstream Akt could phosphorylate KRT19 at S35 and induce localization of KRT19 to the membrane, (3) membrane-localized KRT19 can bind and stabilize HER2 by inhibition of HER2 ubiquitination and (4) KRT19 antibody treatment could interfere with HER2 signaling and reduce proliferation of HER2-positive cells both in vitro and in vivo. A previous study showed that KRT19 was increased in HER2-positive tumors. ${ }^{26}$ They concluded that KRT19 might be responsible for driving the phenotypes of HER2-positive tumors; however, they did not provide any mechanistic relationship between HER2 and KRT19.

It was previously shown that the Sp1-binding site at - 62 is a critical element for KRT19 promoter activity. ${ }^{20}$ In hamster fibroblasts, vascular endothelial growth factor (VEGF) expression is under control of $\mathrm{ERK}^{27}$ which targets $\mathrm{Sp} 1$ and promotes its phosphorylation. ${ }^{11}$ The phosphorylated form of $\mathrm{Sp1}$, in turn, drives transcription from the VEGF promoter, which contains two binding sites for this transcription factor. Sp1 phosphorylation is also achieved by ERK activity. ${ }^{28}$ With these findings, we speculated that HER2/MEK/ERK-driven upregulation of KRT19 transcripts might be mediated by the ERK-dependent activation of Sp1. However, we could not completely rule out the possibility of KRT19 regulation at posttranscriptional levels, as the changes in KRT19 protein level by HER2 is more marked than KRT19 mRNA levels.

KRTs are unique as they are regulated by phosphorylation. $^{29}$ Site-specific phosphorylation occurs during mitosis, where KRTs undergo hyperphosphorylation. ${ }^{29}$ Phosphorylation of KRTs occurs in a particular type of cell to regulate a specific cell function. ${ }^{29}$ In a previous study, KRT19 S35 was reported to be the major phosphorylation site; the same study also showed that a KRT19-S35A mutant exhibits a filament assembly defect. ${ }^{30}$ Our results also indicated that phosphorylation of KRT19 on the S35 residue is an absolute requirement for the transition from a filamentous to a granulous shape. A recent report also showed that Akt2 could induce reorganization of cytoplasmic intermediate filaments including KRTs. ${ }^{31}$ Furthermore, inhibition of tyrosine phosphatase resulted in the formation of KRT13 and KRT5 granules. $^{32}$ Upregulation of p38MAPK activity induced KRT granule formation, whereas pharmacological inhibition of p38MAPK resulted in stabilization of KRT filament networks. ${ }^{33}$ Phosphorylation of residues in the head domain of KRTs were required for the assembly of KRTs. ${ }^{33}$ When hyperphosphorylation status were induced by okadaic acid, KRT18-WT-transfected cells exhibited disassembly of the filament network into short filaments and aggregates of dots, whereas KRT18-S52A-transfected cells exhibited a normal morphology. ${ }^{34}$ On the basis of the findings, we speculated that phosphorylation of S35 could lead to a configuration of KRT19, which might be responsible for the filament disassembly.

Recently, it was reported that full-length KRT19 is released by tumor cells. ${ }^{35}$ The authors showed that KRT19 release is an active process, not a consequence of cell death. As KRT19 lacks both a secretory signal sequence and transmembrane sequence, the authors speculated that the KRT19 release might be mediated by release through exosomes or other unknown transport pathways. They also showed that both MUC1 and KRT19 are co-expressed in the cell periphery, KRT19 staining pattern of which was quite similar to our results shown in Figure 4. On the basis of this previous assumption, we further hypothesized that the translocation mechanism of KRT19 results in its movement to the plasma membrane of HER2-expressing cells (illustrated in Figure 6h). Upon induction by a HER2-downstream MEK/ERK pathway, KRT19 is phosphorylated by Akt on S35. KRT19 phosphorylated on S35 then disassembled into a granulous shape in preparation for transport to the cell membrane or extracellular space, possibly by packaging into exosomes. During its travel to the cell periphery, some KRT19 encounter and bind HER2 inside the trafficking vesicle or near the plasma membrane. Indeed, we also observed that HER2 could increase the release of KRT19 to the culture medium, and treatment with 
MEK, Akt inhibitors or exosome inhibitor 5-( N,N-dimethyl) amiloride hydrochloride could block KRT19 release as well as its binding to HER2 (Supplementary Figure 5). After binding to HER2, KRT19 stabilizes HER2 via inhibition of ubiquitination and subsequent proteosomal degradation of HER2, creating a positive-feedback loop encircling HER2-induced KRT19 expression and KRT19-induced stabilization of HER2. Although elucidation of the precise targeting mechanism of KRT19 to the plasma membrane or extracellular space warrants further experimental challenges, our data indicate that HER2-induced upregulation and subcellular reorganization of KRT19 and subsequent stabilization of HER2, might be responsible for HER2-driven phenotypic changes in cancer cells. We also provide the first evidence that targeting KRT19 by KRT19 antibody could be a novel therapeutic approach for HER2-positive cancer cells, as well as Trastzumab-resistant JIMT-1 cells. ${ }^{36}$ However, it must be noted that complete silencing of KRT19 by shRNA leads to upregulation of cell proliferation by an increase in Akt activity via inhibition of Egr-1/PTEN, as we have reported very recently. ${ }^{37}$ As indicated in Supplementary Figure 6, KRT19 exhibited a biphasic effect on cancer cell proliferation. The cell viability was dependent on KRT19/HER2 signaling at higher KRT19 concentration and it turned to be rather dependent on KRT19/Egr-1 signaling at very low/knockout levels of KRT19.

In conclusion, our studies improve the understanding of HER2-downstream signaling events in cancer cells by demonstrating that HER2-downstream KRT19 could in turn stabilize HER2. Although the usage of KRT19 as a therapeutic target of HER2-positive breast cancer should be carefully considered because of the reason discussed above, our report has implications on using KRT19 as a new therapeutic target against HER2-positve breast cancers.

\begin{abstract}
Materials and Methods
Quantitative mass spectrometry analyses using LC-MS/MS. MCF-7 vec and MCF-7 HER2 cells were prepared as described (Kurokawa et $a^{\beta 8}$ ). MCF-7 vec and MCF-7 HER2 membrane fractions were prepared by centrifugation of cell lysates at $100000 \times g$ in a lysis buffer without detergent. The pellets were first hydrolyzed at aspartic acid using ChemDigest TM (http://www.novacelltech.com). The resulting hydrolysate was digested with trypsin and subjected to chromatographic separation by off-line C18 HPLC and analyzed by LC-MS/MS. Mass analyses were performed using a nano-LC-MS system, which consisted of an Ultimate HPLC system (Agilent 1100, Santa Clara, CA, USA) and a QSTAR quadrupole-TOF mass spectrometer (Applied Biosystems/ MDS SCIEX, Concord, Ontario, Canada) equipped with a nano-ESI source. For MS/MS analysis, each scan cycle consisting of one full-scan mass spectrum ( $\mathrm{m} / \mathrm{z} 400-1500)$ and three MS/MS events were followed. LC-MS/MS data were searched against a concatenate target-decoy database of UniProt v15.15 using MASCOT software (version 2.2.04; Matrix Science, London, UK). The proteins that showed quantitative changes of more than twofold were selected and listed. To estimate fold-changes of identified proteins between experimental groups, we applied label-free quantitative analyses based on comparison of fragment ion intensities. ${ }^{39}$ We got the spectral index (SI) of each protein, which is the cumulative fragment ion intensity for each significantly identified peptide that gives rise to a protein and was normalized (SIN) by dividing the total SI for all identified proteins (SIT). Log2 ratios of normalized SI, Log2 (SIN_HER2/ SIN_vec) were calculated and used as quantitative index. To avoid taking logarithm on zero's, we set the SI as 0.135 that is the 5 th percentile value of SI if no peptide was identified in an experimental group.
\end{abstract}

Cells lines, kinase inhibitors and antibodies. MCF-7 vec, MCF-7 HER2, BT-474, MDA-MB-231, MDA-MB-468, T47D, SKBR-3, JIMT-1 and KPL-4 human breast cancer cells and 293T human kidney cells were maintained in DMEM or RPMI-1640 (HyClone, Logan, UT, USA) supplemented with 10\% fetal bovine serum (FBS), $100 \mathrm{U} / \mathrm{ml}$ penicillin, and $100 \mathrm{mg} / \mathrm{ml}$ streptomycin. KRT19 knockdown cells were generated by transfection with a KRT19 shRNA (Santa Cruz, Santa Cruz, CA, USA) by Lipofectamine 2000 transfection reagent (Invitrogen, Carlsbad, CA, USA). The following kinase inhibitors were used: U0126, LY294002, Wortmannin, Akt inhibitor \#8 and SB203580 (Calbiochem, Darmstadt, Germany); ZD1839 (Biaffin $\mathrm{GmbH} \&$ Co., Kassel, Germany). The following antibodies were used: His, Ubiquitin, KRT1/10 and AQP4 antibodies (Santa Cruz); p-ERK, ERK, p-p38 MAPK, p38 MAPK, Actin, HA, p-HER2, p-Akt and Akt antibodies (Cell Signaling, Beverly, MA USA); KRT19 antibody (Chemicon, Billerica, MA, USA and Santa Cruz); KRT9 antibody (Abcam, Cambridge, MA, USA); KRT18 antibody (Calbiochem); HER2 (ERBB2/neu) antibody (Lab Vision, Fremont, CA, USA); phosphotyrosine antibody (Upstate, Lake Placid, NY, USA); anti-rabbit IgG-Oregon Green (Molecular Probes, Eugene, OR, USA); anti-mouse IgG-Cy3 (Zymed, San Francisco, CA, USA) and anti-Mouse IgG-PE antibody (Abcam).

Plasmids and site-directed mutagenesis. KRT-WT, HER2-WT, HER2CA and HER2-KD were purchased from Addgene (Cambridge, MA, USA). KRT19S10A, and KRT19-S35A were provided by Dr. Omary (Stanford University). The pK19-1970-LucReporter was a gift from Dr. Rustgi (University of Pennsylvania). ERK1-WT, ERK2-WT, p38 MAPK-WT and P38 MAPK-KD were donated by Dr. Su-Jae Lee (Hanyang University). HA-Akt1-CA, HA-Akt1-KD, MEK1-CA and MEK2 were described previously. ${ }^{40,41}$ Using the KRT19-S35A construct as a backbone, KRT19-S10A S35A was produced using a Quickchange kit (Stratagene, San Diego, CA, USA) with the following mutagenic primers: Fwd, 5'-GCTATCGCC AGTCGGCGGCCACGTCGTCCTTC-3', and Rev, 5'-GAAGGACGACGTGGCCGC CGACTGGCGATAGC-3'. Using the HER2-WT construct as a backbone, HER2truncated mutant constructs were produced with the following primers, HER2-FLMyc: Fwd, 5'-cgtaagcttATGGAGCTGGCGG-3', and Rev, 5'-cgttctagaTCACACTG GCACGT-3' (1 to 3768); HER2-ECD-Myc: Fwd, 5'-cgtaagcttATGGAGCTGGCG G-3', and Rev, 5'-cgttctagaGGACGTCAGAGGGC-3' (1 to 1959); HER2-ICD-Myc: Fwd, 5'-cgtaagcttATCATCTCTGCGGTG-3', and Rev, 5'-cgttctagaTCACACTGG CACGT-3' (1960-3768). Hind III and Xbal restriction sites are indicated by lowercase letters. The amplified PCR products were ligated into the pCDNA3.1-myc-His B vector (Invitrogen).

Cell fractionation. PBS-washed cells were pelleted by centrifugation, and cells were broken using a 1-ml syringe ( $26 \mathrm{GX}$ size) in lysis buffer without detergent, after which the supernatants were pelleted by ultracentrifugation for $1 \mathrm{~h}$ at $100000 \times \mathrm{g}$ at $4{ }^{\circ} \mathrm{C}$. After centrifugation, the supernatant was used as the cytosolic fraction and the pellet was resuspended in lysis buffer for use as the membrane fraction.

Reverse transcriptase-PCR (RT-PCR). Expression of transcripts were assessed using the following primers, human KRT19:42 Fwd 5'-GCACTA CAGCCACTACTACACGA-3', Rev 5'-CTCATGCGCAGAGCCTGTT-3', HER2: Fwd 5'-AACTGCACCCACTCCTGTGT-3', Rev 5'-TGATGAGGATCCCAAAGACC-3'. Quantification of actin expression was performed as an internal control using an RT-PCR primer and control set (Invitrogen). Expression of mouse KRT19 was assessed using the following primers: Fwd 5'-TGCTGAAGCCACCTACCTTG-3', Rev 5'-ATACTCCTGGTTCTGGCGCT-3', and mouse actin primers: Fwd 5'-TTCT TTGCTCCTTCGTTGCCG-3', Rev 5'-TGGATGGCTACGTACATGGCTGGG-3'.

Tumor tissue collection. A total of 21 primary invasive breast cancer tissues from different individuals were used to evaluate the expression of KRT19 and HER2 in human breast cancer. Tissue specimens were randomly selected from the tissue archives of the Cancer Research Institute, Seoul National University. All tumors were excised between 2004 and 2006 and were histopathologically confirmed. Informed consent, approved by an Institutional Review Board of Seoul National University Hospital (H-0512-502-163), was obtained for all participants before operation.

Western blot analyses, Immunoprecipitation, Immunofluorescence staining and Immunohistochemistry. For western blot analyses, cells were lysed in a lysis buffer containing $50 \mathrm{mM}$ Tris, pH 8.0, $150 \mathrm{mM} \mathrm{NaCl}$, $1 \%$ NP-40, 0.5\% sodium deoxycholate, $0.1 \%$ SDS, $50 \mathrm{mM} \mathrm{NaF}, 1 \mathrm{mM} \mathrm{Na3VO} 4$ and protease inhibitor cocktail (Roche, Basel, Switzerland). For KRT western blots, TrisTriton buffer containing $10 \mathrm{mM}$ Tris, $\mathrm{pH} 7.4,100 \mathrm{mM} \mathrm{NaCl}, 1 \mathrm{mM}$ EDTA, $1 \mathrm{mM}$ EGTA, 1\% Triton X-100, 10\% glycerol, 0.1\% SDS, 0.5\% deoxycholate (suggested for cytoskeletal proteins by Abcam) was used as a lysis buffer. Samples were 
separated on SDS-PAGE gels and transferred to nitrocellulose membranes (Whatman, Little Chalfont, UK). After blocking with $5 \%$ skim milk in TBST, membranes were incubated with appropriate primary antibodies overnight, followed by $2 \mathrm{~h}$ incubation with HRP-conjugated secondary antibodies. Protein bands were visualized with WEST ZOL plus System (iNtRON, Seongnam, Korea). For Immunoprecipitation, cell lysates $(1 \mathrm{mg})$ were precleared by adding $30 \mu \mathrm{l}$ protein A sepharose (Invitrogen) for $2 \mathrm{~h}$ at $4^{\circ} \mathrm{C}$. After centrifugation at $1000 \times \mathrm{g}$, the supernatant was incubated with either primary antibodies or normal rabbit/mouse immunoglobin $\mathrm{G}(\mathrm{lgG})$ overnight at $4^{\circ} \mathrm{C}$. Protein $\mathrm{A}$ or $\mathrm{G}$ Sepharose $(30 \mu \mathrm{l})$ was added and incubated for $3 \mathrm{~h}$ at $4{ }^{\circ} \mathrm{C}$. After centrifugation, the pellet was washed three times with cell lysis buffer. Immunoprecipitated proteins were resolved on a $10 \%$ SDS-PAGE and subsequently analyzed by western blot. For immunofluorescence staining, cells were washed with PBS, fixed with $4 \%$ paraformaldehyde and permeabilized with $0.1 \%$ Triton X-100 for 15 min. Fixed samples were blocked with $3 \%$ skim milk in PBS for $1 \mathrm{~h}$, followed by incubation with primary antibody diluted in $1 \%$ skim milk in PBS for $1 \mathrm{~h}$. After washing with PBS the samples were treated with the anti-mouse IgG-Cy3 or anti-rabbit IgG-Oregon Green (Molecular Probes). For DNA staining, samples were incubated with Hoechst $33342(1 \mu \mathrm{g} / \mathrm{ml})$ for an additional $10 \mathrm{~min}$. Immunofluorescence was monitored with an Olympus (Tokyo, Japan) upright fluorescence microscope (BX50F) and an Olympus laser scanning confocal microscope (IX2-ILL100). For cell-surface immunofluorescence staining, cells were prepared according to the immunofluorescence staining procedure described above with exception that the permeabilization step was deleted. ${ }^{43}$ For immunohistochemistry, mouse mammary gland tissues were fixed in Bouin's solution (Sigma, St. Louis, MO, USA). Tissues were dehydrated, washed in $70 \%$ ethanol and embedded in paraffin. Five- $\mu$ m-thick paraffin sections were then mounted on poly-L-lysine-coated slides and incubated in a dry oven at $60^{\circ} \mathrm{C}$ for $1 \mathrm{~h}$ followed by deparaffinization in xylene. The slides were then rehydrated in 100, 90 , 80 and $70 \%$ ethanol for 5 min each twice. The slides were incubated with primary antibodies in PBS with $5 \%$ FBS in a humidified chamber overnight. The slides were incubated for $30 \mathrm{~min}$ with a biotin-conjugated secondary antibody (Vector Laboratories, Orton Southgate, UK). The avidin-biotin complex (ABC) reaction was generated according to the manufacturer's protocol. Sections were counterstained with Mayer's hematoxylin (Sigma).

Proliferation assay. For 3-(4,5-dimethylthiazol-2-yl)-2,5-diphenyltetrazolium bromide (MTT) assays, cells were seeded in 96-well culture plates at $5 \times 10^{3}$ cells/well, exposed to indicated antibodies and incubated for $72 \mathrm{~h}$. Cell viability was assessed by adding $20 \mu \mathrm{l}$ of $10 \mathrm{mg} / \mathrm{ml}$ MTT (Sigma) to $100 \mu \mathrm{l}$ of culture medium, and incubating for $3 \mathrm{~h}$ at $37^{\circ} \mathrm{C}$ in a $\mathrm{CO}_{2}$ incubator. The medium was removed, formazan was dissolved in DMSO (Sigma), and the optical density was measured at $590 \mathrm{~nm}$ using a Multiskan EX (Thermo, Waltham, MA, USA).

In vitro kinase assay. GST expression plasmids expressing were transformed into E. coli BL21 (DE3). Growth of the E. coli was then induced by addition of $1 \mathrm{mM}$ isopropyl 1-thio- $\beta$-D-galactopyranoside, and after $4 \mathrm{~h}$ the cultures were collected. Cells were then lysed with a sonicator. Supernatants were loaded onto a glutathione Sepharose 4B column (GE Healthcare, Piscataway, NJ, USA), and bound GSTtagged proteins were eluted in elution buffer $(50 \mathrm{mM}$ Tris- $\mathrm{HCl} \mathrm{pH} \mathrm{9.5,10 \textrm {mM }}$ reduced glutathione). $293 \mathrm{~T}$ cells seeded at the density of $1 \times 10^{6}$ total cells per 100 $\mathrm{mm}$ dish were transfected with $10 \mu \mathrm{g}$ of HA-Akt and collected $48 \mathrm{~h}$ after transfection. Next, a total of $1 \mathrm{mg}$ of whole-cell lysate was incubated with HA-antibody and subsequently treated with protein A-Sepharose CL-4B. The resulting immunocomplexes were incubated with $1.5 \mu \mathrm{g}$ of pre-purified GST-KRT19 in $15 \mu \mathrm{l}$ kinase reaction buffer ( $25 \mathrm{mM}$ Tris- $\mathrm{HCl}$ (pH 7.5), $5 \mathrm{mM} \beta$-glycerophosphate, $0.1 \mathrm{mM}$ sodium orthovanadate, $2 \mathrm{mM}$ dithiothreitol, $200 \mu \mathrm{M}$ ATP, $10 \mathrm{mM}$ magnesium chloride) and $10 \mu \mathrm{Ci}[\gamma 32 \mathrm{P}]$ ATP (GE Healthcare) for $30 \mathrm{~min}$ at RT. Samples containing 32Plabeled proteins were analyzed by SDS-PAGE and subsequent autoradiography.

Cycloheximide-decay assay. At $48 \mathrm{~h}$ post transfection in $293 \mathrm{~T}, 20 \mu \mathrm{g} / \mathrm{ml}$ cycloheximide (Sigma) was added to block further protein synthesis. MCF-7 cells were treated with $100 \mu \mathrm{g} / \mathrm{ml}$ cycloheximide. Cells were collected at each of the indicated time points and subjected to western blot analysis.

Ubiquitination assay. We transfected 293T cells with a His-ubiquitin expression construct with the indicated plasmids. At $36 \mathrm{~h}$ post transfection, cells were treated with the proteosomal inhibitor MG132, $10 \mu \mathrm{M}$ for $8 \mathrm{~h}$. Cells were then collected and lysed. The supernatant was incubated with $4 \mu \mathrm{g}$ of His antibody overnight at $4{ }^{\circ} \mathrm{C}$. Protein A sepharose $(30 \mu \mathrm{l})$ was then added and incubated for $4 \mathrm{~h}$ at $4{ }^{\circ} \mathrm{C}$. After centrifugation at $1000 \times g$, the pellet was washed three times with lysis buffer. The immunoprecipitated proteins were resolved by SDS-PAGE and subjected to western blot analysis.

Dual luciferase assays. MCF-7 cells in 12-well plates were transfected with $0.2 \mu \mathrm{g}$ of reporter constructs, pK19-1970-LucReporter, ${ }^{20}$ and $0.002 \mu \mathrm{g}$ of pCMV-RI as an internal control by using Lipofectamine 2000 (Invitrogen). At $48 \mathrm{~h}$ post transfection, dual luciferase assays were performed according to manufacturer's protocol (Promega, Madison, WI, USA). 293T cells were transfected with $1 \mu \mathrm{g}$ total DNA containing $500 \mathrm{ng}$ of pK19-1970-LucReporter and $0.002 \mu \mathrm{g}$ of pCMV-RI constructs by calcium phosphate transfection method.

In vivo labeling. 293T cells were transfected each with $4 \mu \mathrm{g} \mathrm{HA-Akt1-CA}$ and $\mathrm{KRT} 19$, and labeled with $500 \mu \mathrm{Ci} / \mathrm{mL}$ of [32P] orthophosphate for $18 \mathrm{~h}$ in phosphatefree DMEM containing $10 \%$ dialyzed FBS. Total cell lysates $(1 \mathrm{mg}$ ) were precleared by adding $30 \mu \mathrm{l}$ protein A sepharose (Invitrogen) for $2 \mathrm{~h}$ at $4{ }^{\circ} \mathrm{C}$. After centrifugation at $1000 \times g$, the supernatant was incubated with $4 \mu \mathrm{g}$ of KRT19 antibody overnight at $4{ }^{\circ} \mathrm{C}$. Protein $\mathrm{A}$ sepharose $(30 \mu \mathrm{l})$ was then added and incubated for $4 \mathrm{~h}$ at $4{ }^{\circ} \mathrm{C}$. After centrifugation at $1000 \times g$, the pellet was washed three times with lysis buffer. Finally, the immunoprecipitated proteins were resolved by SDS-PAGE and subsequent autoradiography.

Cell surface protein biotinylation assay. Cell surface protein biotinylation assay was performed according to Wu et al..$^{44}$ In brief, 80 90\% confluence cells in 100-mm dishes were washed twice with ice-cold PBS and incubated for a further $15 \mathrm{~min}$ in ice-cold PBS. Biotinylation was performed by incubating cells in PBS containing $0.5 \mathrm{mg} / \mathrm{ml}$ of NHS-SS-Biotin (Thermo) for $30 \mathrm{~min}$ at $4^{\circ} \mathrm{C}$. To determine cell-surface level of KRT19, labeled cells were lysed, precipitated with streptavidin-conjugated agarose beads (Invitrogen), and subjected to western blotting with KRT19 antibody. For negative control of non-specific labeling of internal proteins, biotin-labeled cells were washed by PBS twice, then washed by glutathione (GSH) (Sigma).

KRT19 and mouse normal IgG antibodies. We purchased a custombuilt hybridoma cells for mass production of monoclonal KRT19 antibodies from Abby mart Biomedical Co. Ltd. Shanghai (Shanghai, China). Mouse myeloma cells, TIB-9 were purchased from ATCC (Manassas, VA, USA). Antibody production was performed according to Yokoyama et al. ${ }^{45}$ In brief, at least 6-week-old BALB/C mice were i.p. injected pristine (Sigma). After a week, mice were inoculated with $1 \times 10^{6}$ hybridoma and cells suspended in incomplete Freund's adjuvant (Sigma). Ascites fluid was collected using 18-gauge needles. Antibodies were purified by Montage antibody purification kit (Millipore, Billerica, MA, USA) and screened for endotoxin test.

Tumor growth model and antitumor activity of KRT19 antibody in vivo. Eight-week-old BALB/C nude mice were purchased from ORIENT BIO Inc (Seongnam, Korea). The mice were cared for and used in compliance with institutional guidelines. The mice were given a single s.c. injection of $5 \times 10^{6} \mathrm{KPL}-4$ cells suspended in PBS. Tumors were allowed to grow until they reached a diameter of 3-6 $\mathrm{mm}$ (day 0 ). The mice were then randomly divided into different treatment groups. Trastzumab (Roche), KRT19 antibody and mouse nomal IgG were i.p. injected at a dose of $20 \mathrm{mg} / \mathrm{kg}$ for every 3 days. Tumor volumes in $\mathrm{mm}^{3}$ was calculated by the formula: volume $=(\text { width })^{2} \times$ length $/ 2$.

\section{Conflict of Interest}

The authors declare no conflict of interest.

Acknowledgements. This work was supported by NRF grant (2013-059143) from the Korea Research Foundation and the Converging Research Center Program funded by Ministry of Science, ICT \& Future Planning (Project No. 2014048814). We thank Dr. Omary (Stanford University), Dr. Rustgi (Univ of Pennsylvania) and Dr. Su-Jae Lee for the plasmid constructs.

1. Slamon DJ, Clark GM, Wong SG, Levin WJ, Ullrich A, McGuire WL. Human breast cancer: correlation of relapse and survival with amplification of the HER-2/neu oncogene. Science 1987; 235: 177-182. 
2. Tzahar E, Waterman H, Chen X, Levkowitz G, Karunagaran D, Lavi S et al. A hierarchical network of interreceptor interactions determines signal transduction by $\mathrm{Neu}$ differentiation factor/neuregulin and epidermal growth factor. Mol Cell Biol 1996; 16: 5276-5287.

3. Karunagaran D, Tzahar E, Beerli RR, Chen X, Graus-Porta D, Ratzkin BJ et al. ErbB-2 is a common auxiliary subunit of NDF and EGF receptors: implications for breast cancer. EMBO J 1996; 15: 254-264.

4. Neve RM, Sutterluty $H$, Pullen $N$, Lane HA, Daly JM, Krek W et al. Effects of oncogenic ErbB2 on G1 cell cycle regulators in breast tumour cells. Oncogene 2000; 19: 1647-1656.

5. Munster PN, Marchion DC, Basso AD, Rosen N. Degradation of HER2 by ansamycins induces growth arrest and apoptosis in cells with HER2 overexpression via a HER3, phosphatidylinositol 3'-kinase-AKT-dependent pathway. Cancer Res 2002; 62: 3132-3137.

6. Manning BD, Cantley LC. AKT/PKB signaling: navigating downstream. Cell 2007; 129: 1261-1274.

7. Alessi DR, Caudwell FB, Andjelkovic M, Hemmings BA, Cohen P. Molecular basis for the substrate specificity of protein kinase B; comparison with MAPKAP kinase-1 and p70 S6 kinase. FEBS Lett 1996; 399: 333-338

8. Gonzalez FA, Seth A, Raden DL, Bowman DS, Fay FS, Davis RJ. Serum-induced translocation of mitogen-activated protein kinase to the cell surface ruffling membrane and the nucleus. J Cell Biol 1993; 122: 1089-1101.

9. Marais R, Wynne J, Treisman R. The SRF accessory protein Elk-1 contains a growth factorregulated transcriptional activation domain. Cell 1993; 73: 381-393.

10. Okazaki K, Sagata N. The Mos/MAP kinase pathway stabilizes c-Fos by phosphorylation and augments its transforming activity in NIH 3T3 cells. EMBO J 1995; 14: 5048-5059.

11. Milanini-Mongiat J, Pouyssegur J, Pages G. Identification of two Sp1 phosphorylation sites for p42/p44 mitogen-activated protein kinases: their implication in vascular endothelial growth factor gene transcription. J Biol Chem 2002; 277: 20631-20639.

12. Plowman JE. The proteomics of keratin proteins. J Chromatogr B Analyt Technol Biomed Life Sci 2007; 849: 181-189.

13. Coulombe PA, Wong P. Cytoplasmic intermediate filaments revealed as dynamic and multipurpose scaffolds. Nat Cell Biol 2004; 6: 699-706.

14. Wu YJ, Rheinwald JG. A new small (40 kd) keratin filament protein made by some cultured human squamous cell carcinomas. Cell 1981; 25: 627-635.

15. Bader BL, Magin TM, Hatzfeld M, Franke WW. Amino acid sequence and gene organization of cytokeratin no. 19, an exceptional tail-less intermediate filament protein. EMBO J 1986; 5: $1865-1875$.

16. Ignatiadis M, Xenidis N, Perraki M, Apostolaki S, Politaki E, Kafousi M et al. Different prognostic value of cytokeratin-19 mRNA positive circulating tumor cells according to estrogen receptor and HER2 status in early-stage breast cancer. J Clin Oncol 2007; 25: 5194-5202.

17. Bozionellou V, Mavroudis D, Perraki M, Papadopoulos S, Apostolaki S, Stathopoulos E et al. Trastuzumab administration can effectively target chemotherapy-resistant cytokeratin-19 messenger RNA-positive tumor cells in the peripheral blood and bone marrow of patients with breast cancer. Clin Cancer Res 2004; 10: 8185-8194.

18. Xenidis N, Vlachonikolis I, Mavroudis D, Perraki M, Stathopoulou A, Malamos N et al. Peripheral blood circulating cytokeratin-19 mRNA-positive cells after the completion of adjuvant chemotherapy in patients with operable breast cancer. Ann Oncol 2003; 14: 849-855.

19. Park S, Lee KM, Ju JH, Kim J, Noh DY, Lee T et al. Protein expression profiling of primary mammary epithelial cells derived from MMTV-neu mice revealed that HER2/NEU-driven changes in protein expression are functionally clustered. IUBMB Life 2010; 62: 41-50.

20. Brembeck FH, Rustgi AK. The tissue-dependent keratin 19 gene transcription is regulated by GKLF/KLF4 and Sp1. J Biol Chem 2000; 275: 28230-28239.

21. Johnson JL, Pacquelet S, Lane WS, Eam B, Catz SD. Akt regulates the subcellular localization of the Rab27a-binding protein JFC1 by phosphorylation. Traffic 2005; 6 : 667-681.

22. Basu S, Totty NF, Irwin MS, Sudol M, Downward J. Akt phosphorylates the Yes-associated protein, YAP, to induce interaction with 14-3-3 and attenuation of p73-mediated apoptosis. Mol Cell 2003; 11: 11-23.

23. Fang D, Hawke D, Zheng Y, Xia Y, Meisenhelder J, Nika $\mathrm{H}$ et al. Phosphorylation of betacatenin by AKT promotes beta-catenin transcriptional activity. J Biol Chem 2007; 282 11221-11229.
24. Hoyal CR, Gutierrez A, Young BM, Catz SD, Lin JH, Tsichlis PN et al. Modulation of p47PHOX activity by site-specific phosphorylation: Akt-dependent activation of the NADPH oxidase. Proc Natl Acad Sci USA 2003; 100: 5130-5135.

25. Zhang H, Zha X, Tan Y, Hornbeck PV, Mastrangelo AJ, Alessi DR et al. Phosphoprotein analysis using antibodies broadly reactive against phosphorylated motifs. J Biol Chem 2002; 277: 39379-39387.

26. Zhang DH, Tai LK, Wong LL, Sethi SK, Koay ES. Proteomics of breast cancer: enhanced expression of cytokeratin19 in human epidermal growth factor receptor type 2 positive breast tumors. Proteomics 2005; 5: 1797-1805.

27. Milanini J, Vinals F, Pouyssegur J, Pages G. p42/p44 MAP kinase module plays a key role in the transcriptional regulation of the vascular endothelial growth factor gene in fibroblasts. J Biol Chem 1998; 273: 18165-18172.

28. Benasciutti E, Pages G, Kenzior O, Folk W, Blasi F, Crippa MP. MAPK and JNK transduction pathways can phosphorylate $\mathrm{Sp} 1$ to activate the UPA minimal promoter element and endogenous gene transcription. Blood 2004; 104: 256-262.

29. Omary MB, Ku NO, Tao GZ, Toivola DM, Liao J. "Heads and tails" of intermediate filament phosphorylation: multiple sites and functional insights. Trends Biochem Sci 2006; 31: 383-394.

30. Zhou X, Liao J, Hu L, Feng L, Omary MB. Characterization of the major physiologic phosphorylation site of human keratin 19 and its role in filament organization. $J$ Biol Chem 1999; 274: 12861-12866.

31. Fortier AM, Van Themsche C, Asselin E, Cadrin M. Akt isoforms regulate intermediate filament protein levels in epithelial carcinoma cells. FEBS Lett 2010; 584: 984-988.

32. Strnad $P$, Windoffer $R$, Leube RE. Induction of rapid and reversible cytokeratin filament network remodeling by inhibition of tyrosine phosphatases. J Cell Sci 2002; 115: 4133-4148.

33. Woll S, Windoffer R, Leube RE. p38 MAPK-dependent shaping of the keratin cytoskeleton in cultured cells. J Cell Biol 2007; 177: 795-807.

34. Ku NO, Omary MB. Identification of the major physiologic phosphorylation site of human keratin 18: potential kinases and a role in filament reorganization. J Cell Biol 1994; 127: $161-171$.

35. Alix-Panabieres C, Vendrell JP, Slijper M, Pelle O, Barbotte E, Mercier G et al. Full-length cytokeratin-19 is released by human tumor cells: a potential role in metastatic progression of breast cancer. Breast Cancer Res 2009; 11: R39.

36. Nagy $\mathrm{P}$, Friedlander $\mathrm{E}$, Tanner M, Kapanen Al, Carraway $\mathrm{KL}$, Isola $\mathrm{J}$ et al. Decreased accessibility and lack of activation of ErbB2 in JIMT-1, a herceptin-resistant, MUC4expressing breast cancer cell line. Cancer Res 2005; 65: 473-482.

37. Ju JH, Yang W, Lee KM, Oh S, Nam K, Shim S et al. Regulation of cell proliferation and migration by keratin19-induced nuclear import of early growth response-1 in breast cancer cells. Clin Cancer Res 2013; 19: 4335-4346.

38. Kurokawa H, Lenferink AE, Simpson JF, Pisacane PI, Sliwkowski MX, Forbes JT et al. Inhibition of HER2/neu (erbB-2) and mitogen-activated protein kinases enhances tamoxifen action against HER2-overexpressing, tamoxifen-resistant breast cancer cells. Cancer Res 2000; 60: 5887-5894.

39. Griffin NM, Yu J, Long F, Oh P, Shore S, Li Y et al. Label-free, normalized quantification of complex mass spectrometry data for proteomic analysis. Nat Biotechnol 2010; 28: 83-89.

40. Abbott DW, Holt JT. Mitogen-activated protein kinase kinase 2 activation is essential for progression through the $\mathrm{G} 2 / \mathrm{M}$ checkpoint arrest in cells exposed to ionizing radiation. J Biol Chem 1999; 274: 2732-2742.

41. Shin I, Yakes FM, Rojo F, Shin NY, Bakin AV, Baselga J et al. PKB/Akt mediates cell-cycle progression by phosphorylation of p27(Kip1) at threonine 157 and modulation of its cellular localization. Nat Med 2002; 8: 1145-1152.

42. Stathopoulou A, Gizi A, Perraki M, Apostolaki S, Malamos N, Mavroudis D et al. Real-time quantification of CK-19 mRNA-positive cells in peripheral blood of breast cancer patients using the lightcycler system. Clin Cancer Res 2003; 9: 5145-5151.

43. Roychowdhury S, Vyas PM, Reilly TP, Gaspari AA, Svensson CK. Characterization of the formation and localization of sulfamethoxazole and dapsone-associated drug-protein adducts in human epidermal keratinocytes. J Pharmacol Exp Ther 2005; 314: 43-52.

44. Wu X, Gan B, Yoo Y, Guan JL. FAK-mediated src phosphorylation of endophilin A2 inhibits endocytosis of MT1-MMP and promotes ECM degradation. Dev Cell 2005; 9: 185-196.

45. Yokoyama WM. Production of monoclonal antibody supernatant and ascites fluid. Curr Protoc Mol Biol 2008; Chapter 11: Unit 1110. 